\title{
SOLVING SINGULAR GENERALIZED EIGENVALUE PROBLEMS BY A RANK-COMPLETING PERTURBATION
}

\author{
MICHIEL E. HOCHSTENBACH* ${ }^{*}$, CHRISTIAN MEHL ${ }^{\dagger}$, AND BOR PLESTENJAK ${ }^{\ddagger}$
}

\begin{abstract}
Generalized eigenvalue problems involving a singular pencil are very challenging to solve, both with respect to accuracy and efficiency. The existing package Guptri is very elegant but may be time-demanding, even for small and medium-sized matrices. We propose a simple method to compute the eigenvalues of singular pencils, based on one perturbation of the original problem of a certain specific rank. For many problems, the method is both fast and robust. This approach may be seen as a welcome alternative to staircase methods.
\end{abstract}

Key words. Singular pencil, singular generalized eigenvalue problem, rank-completing perturbation, Guptri, model updating, double eigenvalues, two-parameter eigenvalue problem, differential algebraic equations, quadratic two-parameter eigenvalue problem.

AMS subject classifications. 65F15, 15A18, 15A22, 15A21, 47A55, 65F22

1. Introduction. We study the computation of eigenvalues of small to medium-sized matrix pencils $A-\lambda B$, where $A$ and $B$ are (real or complex) $n \times m$ matrices such that the matrix pencil $A-\lambda B$ is singular, which means that $m \neq n$, or if $m=n$ then

$$
\operatorname{det}(A-\lambda B) \equiv 0
$$

In these cases, the common definition of eigenvalues as roots of $\operatorname{det}(A-\lambda B)$ would only be meaningful for the case $m=n$, but turns out to be useless as any value $\lambda \in \mathbb{C}$ would be an eigenvalue. Therefore, finite eigenvalues of a singular matrix pencil $A-\lambda B$ are typically defined as values $\lambda_{0} \in \mathbb{C}$ satisfying $\operatorname{rank}\left(A-\lambda_{0} B\right)<\operatorname{nrank}(A, B)$, where

$$
\operatorname{nrank}(A, B):=\max _{\zeta \in \mathbb{C}} \operatorname{rank}(A-\zeta B)
$$

denotes the normal rank of the pencil $A-\lambda B$; see [10]. Similarly, we say that $\infty$ is an eigenvalue of the singular pencil $A-\lambda B$ if $\operatorname{rank}(B)<\operatorname{nrank}(A, B)$. In the following we will mainly restrict ourselves to the case $m=n$ as the case $m \neq n$ can easily be reduced to the square case by adding an appropriate number of zero rows or columns.

The singular generalized eigenvalue problem (singular GEP) is well known to be ill-conditioned as arbitrarily small perturbation may cause drastic changes in the eigenvalues. A classical example is given by the pencils

$$
A-\lambda B=\left[\begin{array}{ll}
1 & 0 \\
0 & 0
\end{array}\right]-\lambda\left[\begin{array}{ll}
1 & 0 \\
0 & 0
\end{array}\right] \text { and } \widetilde{A}-\lambda \widetilde{B}=\left[\begin{array}{cc}
1 & \varepsilon_{1} \\
\varepsilon_{2} & 0
\end{array}\right]-\lambda\left[\begin{array}{cc}
1 & \varepsilon_{3} \\
\varepsilon_{4} & 0
\end{array}\right]
$$

where $\varepsilon_{1}, \ldots, \varepsilon_{4} \in \mathbb{C} \backslash\{0\}$; see [22]. While $A-\lambda B$ is singular and has only the eigenvalue 1 , the perturbed pencil $\widetilde{A}-\lambda \widetilde{B}$ is regular and has the eigenvalues $\frac{\varepsilon_{1}}{\varepsilon_{3}}$ and $\frac{\varepsilon_{2}}{\varepsilon_{4}}$ that can be anywhere in the complex plane even for tiny absolute values of $\varepsilon_{1}, \ldots, \varepsilon_{4}$.

${ }^{*}$ Version April 3, 2019. Department of Mathematics and Computer Science, TU Eindhoven, PO Box 513,5600 MB, The Netherlands, www.win.tue.nl/ hochsten. This author has been supported by an NWO Vidi research grant.

${ }^{\dagger}$ Institut für Mathematik, Technische Universität Berlin, Sekretariat MA 4-5, Straße des 17. Juni 136, 10623 Berlin, Germany, mehl@math.tu-berlin.de. This author has been supported by a Dutch 4TU AMI visitor's grant.

${ }^{\ddagger}$ IMFM and Faculty of Mathematics and Physics, University of Ljubljana, Jadranska 19, SI-1000 Ljubljana, Slovenia, bor.plestenjak@fmf.uni-lj.si. This author has been supported in part by the Slovenian Research Agency (grant P1-0294). 
On the other hand, it was observed in [43] that a situation as above is exceptional and that generically small perturbations of a singular square pencil make the pencil regular and some of the eigenvalues of the perturbed pencil are very close to the original eigenvalues of the singular pencil. The following example illustrates this. The Matlab commands

$$
\begin{aligned}
& A=\operatorname{diag}\left(\left[\begin{array}{llllll}
1 & 2 & 3 & 0 & 0 & 0
\end{array}\right]\right) ; \\
& B=\operatorname{diag}\left(\left[\begin{array}{llllll}
2 & 3 & 4 & 0 & 0 & 0
\end{array}\right]\right) ; \\
& \text { eig }\left(U^{\prime} * A * V, \quad U^{\prime} * B * V\right)
\end{aligned}
$$

where $U$ and $V$ are certain random $6 \times 6$ orthogonal matrices, compute the following eigenvalues:

$$
\begin{array}{llllll}
0.5000 & 0.6667 & 0.7500 & 0.1595 & 0.6756 & 0.6543
\end{array}
$$

We see that the three (finite) eigenvalues of the regular part are correct. Following the terminology of [40], the other three values are "fake eigenvalues" and correspond to the singular part of the pencil. (Explicit error analysis for the eigenvalues of singular pencils has been undertaken in [9, 11.) Despite this observation, Van Dooren suggests in [40] to solve the singular generalized eigenvalue problem by first extracting the regular part and then use the QZ algorithm on that part. Wilkinson strongly supports this recommendation in [43].

A robust software package which follows Van Dooren's approach is Guptri [13, 17. For a singular pencil, first a "staircase" algorithm is applied to deflate the singular part of the pencil, and then the QZ algorithm is used to compute the eigenvalues of the remaining regular part. While the results of Guptri are usually excellent, this method may be quite time-consuming; for instance, applying Guptri on a singular $300 \times 300$ pencil on our machine took over 20 seconds, while Matlab's eig on a random pencil of the same size spent less than a second 1 Another issue is the fact that staircase type methods such as Guptri need rank decisions. If the pencil has a minimal index of size $\eta$ (see Section 2 for more details), then at least $\eta+1$ such decisions have to be taken. Typically, these decisions tend to become more and more critical during a run of the staircase algorithm. See, e.g., [14] or [33, Ex. 18], where a variant of the staircase algorithm for the singular two-parameter eigenvalue problem, introduced in [31], fails in double precision but gets the right result in higher precision.

Another way of extracting the regular part using fewer rank decisions has been suggested in [30]. One may view the singular pencil as a constant coefficient differential-algebraic equation and perform a regularization procedure with the help of a derivative array as described in [3]. In this way, the regular part of the pencil can be extracted by only three nullspace computations. However, the derivative array approach leads to an inflation of the system by a factor of at least $\eta+1$, where $\eta$ is the largest minimal index of the given pencil, and may thus result in high computational costs.

We propose a new method to compute the eigenvalues of a singular pencil. The method is based on considering perturbations of rank

$$
k=n-\operatorname{nrank}(A, B)
$$

which we will call rank-completing perturbations as the rank is exactly large enough to generically turn the pencil into a pencil of full normal rank. As we will show, the canonical form of the original regular part of the given pencil stays invariant under generic rank-completing perturbations.

The idea of computing eigenvalues of singular pencils with rank-completing perturbations is not completely new, and the following specific type has been used in system theory as early as in the 70s (without the use of the terminology "rank-completing perturbation"). If a linear

\footnotetext{
${ }^{1}$ We note that this experiment has been performed some years ago. A current practical issue is that there is no publicly available 64-bit Guptri code.
} 
time-invariant control system of the form

$$
\begin{aligned}
& \dot{x}=A x+B u, \\
& y=C x+D u
\end{aligned}
$$

is given, where $A \in \mathbb{R}^{n, n}, B \in \mathbb{R}^{n, m}, C \in \mathbb{R}^{r, n}$, and $D \in \mathbb{R}^{r, m}$ are the system matrices, $x$ stands for the state of the system, $u$ is the input, and $y$ is the output, then the eigenvalues of the system pencil

$$
S(\lambda)=\left[\begin{array}{cc}
\lambda I-A & B \\
-C & D
\end{array}\right]
$$

are of particular interest in control theory; see [15] and the references therein. (If the system is minimal, then these eigenvalues are also referred to as transmission zeros of the system.) Clearly, if $m \neq r$, then the pencil $S(\lambda)$ is rectangular and thus singular. For that case and under the additional assumptions $r<m$ and nrank $S(\lambda)=n+r$, the following algorithm based on ideas of [6] has been proposed in [24] for the computation of the transmission zeros:

1: $\quad$ Select random matrices $\left[\begin{array}{ll}E_{1} & F_{1}\end{array}\right],\left[\begin{array}{ll}E_{2} & F_{2}\end{array}\right] \in \mathbb{R}^{m-r, n+m}$ so that

$$
S_{i}(\lambda):=\left[\begin{array}{cc}
\lambda I-A & B \\
-C & D \\
E_{i} & F_{i}
\end{array}\right]
$$

is regular for $i=1,2$.

2: $\quad$ Compute the eigenvalues $\mathcal{E}_{i}$ of $S_{i}(\lambda)$ for $i=1,2$.

3: Compute the intersection $\mathcal{E}=\mathcal{E}_{1} \cap \mathcal{E}_{2}$.

Since for each eigenvalue $\lambda_{0}$ of $S(\lambda)$ we have $\operatorname{rank} S_{i}\left(\lambda_{0}\right)<n+m$, it immediately follows that the eigenvalues of $S(\lambda)$ are contained in the spectrum of $S_{i}(\lambda)$ for both $i=1,2$. The extended matrix pencils will give rise to two sets of fake eigenvalues. As generically these sets will be disjoint if the applied perturbations are generated randomly, it follows that the set $\mathcal{E}$ will generically coincide with the set of eigenvalues of $S(\lambda)$.

However, as pointed out in [15], this method may encounter difficulties in distinguishing the finite zeros from the infinite ones, in particular if the latter occur with a high multiplicity. Another problem may occur in identifying the values that belong to the intersection $\mathcal{E}$. Although the original eigenvalues of the pencil theoretically coincide with a subset of both $\mathcal{E}_{1}$ and $\mathcal{E}_{2}$, they may still differ slightly in practice due to finite precision arithmetic. Therefore, a tolerance has to be prescribed that decides when two values are considered to be equal. If this tolerance is chosen too small, then some of the eigenvalues may be missed. If, on the other hand, the tolerance is set too large, then two close fake eigenvalues of $S_{1}(\lambda)$ and $S_{2}(\lambda)$ may be falsely identified as an eigenvalue of $S(\lambda)$.

In this paper, we show that the eigenvalues of a singular pencil can be efficiently computed with the help of just one rank-completing perturbation of the form

$$
A-\lambda B+\tau U\left(D_{A}-\lambda D_{B}\right) V^{*}
$$

where $U, V$ are $n \times k$ matrices with orthonormal columns, $D_{A}, D_{B}$ are diagonal $k \times k$ matrices, and $\tau$ is a nonzero scalar. The orthonormality of the columns is not strictly necessary, but convenient, for instance since in this case the norm of the perturbation can easily be controlled by the parameter $\tau$. The problem of identifying the subset of eigenvalues of the original pencil among the computed eigenvalues of the perturbed pencil is then taken care of by the key observation that the left and right eigenvectors that correspond to the true eigenvalues satisfy orthogonality relations 
with respect to the matrices $U$ and $V$. Thus, instead of comparing the spectra of two different pencils, the true eigenvalues can be separated from the fake eigenvalues by using information from the corresponding left and right eigenvectors from only one perturbed pencil. We note that perturbations of singular matrix pencils have already been considered in [4, 9, 27, 38, 39], but it seems that a detailed investigation of rank-completing perturbations is new, except for [28], where the case of singular Hermitian pencils of normal rank $n-1$ was considered.

The rest of this paper is organized as follows. After some preliminaries in Section 2, we review some motivating applications where one is interested in computing eigenvalues of a singular matrix pencil in Section 3. The main theoretical results are presented in Section 4, while the numerical method based on these results is introduced in Section 5, followed by some numerical experiments in Section 6. In Section 7 we discuss singular two-parameter eigenvalue problems and present a new numerical method for such problems. We summarize some conclusions in Section 8 .

2. Preliminaries. We will interpret matrix pencils both as pairs of matrices $(A, B) \in \mathbb{C}^{n, m} \times$ $\mathbb{C}^{n, m}$ or as $n \times m$ matrix polynomials $A-\lambda B$ of degree at most one and we will switch between these notations whenever useful. An important tool in the theory of singular pencils is the Kronecker canonical form (KCF) of a pencil $A-\lambda B$; see, e.g., [16].

Theorem 2.1 (Kronecker canonical form). Let $A-\lambda B$ be a complex $n \times m$ matrix pencil. Then there exist nonsingular matrices $P \in \mathbb{C}^{n, n}$ and $Q \in \mathbb{C}^{m, m}$ such that

$$
P(A-\lambda B) Q=\left[\begin{array}{cc}
R(\lambda) & 0 \\
0 & S(\lambda)
\end{array}\right], \quad R(\lambda)=\left[\begin{array}{cc}
J-\lambda I_{r} & 0 \\
0 & I_{s}-\lambda N
\end{array}\right]
$$

with $J, N$ in Jordan normal form and in addition $N$ being nilpotent, and

$$
S(\lambda)=\operatorname{diag}\left(L_{m_{1}}(\lambda), \ldots, L_{m_{k}}(\lambda), L_{n_{1}}(\lambda)^{\top}, \ldots, L_{n_{\ell}}(\lambda)^{\top}\right)
$$

where $L_{j}(\lambda)=\left[\begin{array}{ll}0 & I_{j}\end{array}\right]-\lambda\left[\begin{array}{ll}I_{j} & 0\end{array}\right]$ is of size $j \times(j+1)$, and $m_{i} \geq 0$ for $i=1, \ldots, k$, and $n_{i} \geq 0$ for $i=1, \ldots, \ell$.

The pencils $R(\lambda)$ and $S(\lambda)$ in Theorem 2.1 are called the regular and the singular part of $A-\lambda B$, respectively. The eigenvalues of $J$ are exactly the finite eigenvalues of $A-\lambda B$, while the eigenvalue 0 of $N$ corresponds to the infinite eigenvalue of $A-\lambda B$. The parameters $m_{1}, \ldots, m_{k}$ and $n_{1}, \ldots, n_{\ell}$ are called the right and the left minimal indices of $A-\lambda B$, respectively. One may easily check that the normal $\operatorname{rank} \operatorname{nrank}(A, B)$ is equal to $\min (n-\ell, m-k)$. In the remainder of the paper, we will consider square pencils $A-\lambda B$, i.e., we have $n=m$. Note that this implies $k=\ell$, i.e., we must have the same number of right and left minimal indices. However, the particular values of the left and right minimal indices may be distinct.

In contrast to the eigenvalues of singular pencils, the corresponding eigenvectors and deflating subspaces are not well defined. To understand why, we consider the following example borrowed from [29] (and slightly adapted). The pencil

$$
A-\lambda B=\left[\begin{array}{crc}
1-\lambda & 0 & 0 \\
0 & -\lambda & 1 \\
0 & 0 & 0
\end{array}\right]
$$

obviously has the regular part $R(\lambda)=[1-\lambda]$ and thus the pencil $A-\lambda B$ has the single eigenvalue $\lambda_{0}=1$ with algebraic multiplicity one. Nevertheless, any vector of the form $x(\alpha, \beta):=\left[\begin{array}{lll}\alpha \beta & \beta\end{array}\right]^{\top}$ with $\alpha, \beta \in \mathbb{C}$ satisfies $A x=\lambda_{0} B x$ and thus could be interpreted as an eigenvector of the pencil. One may argue that the choice $\alpha \neq 0$ and $\beta=0$ seems to be canonical and gives a unique 
one-dimensional deflating subspace "corresponding" to the regular part of the pencil. But on the other hand it follows from the equality

$$
\left[\begin{array}{ccc}
1 / \alpha & 0 & 0 \\
-\beta / \alpha & 1 & 0 \\
0 & 0 & 1
\end{array}\right]\left[\begin{array}{crc}
1-\lambda & 0 & 0 \\
0 & -\lambda & 1 \\
0 & 0 & 0
\end{array}\right]\left[\begin{array}{lll}
\alpha & 0 & 0 \\
\beta & 1 & 0 \\
\beta & 0 & 1
\end{array}\right]=\left[\begin{array}{rrr}
1-\lambda & 0 & 0 \\
0 & -\lambda & 1 \\
0 & 0 & 0
\end{array}\right]
$$

that for any choice of $\alpha, \beta$ with $\alpha \neq 0$, the vector $x(\alpha, \beta)$ can be used to extract the regular part of the pencil as well (and thus could also be considered as "corresponding" to the regular part). For this reason, we restrict ourselves to the computation of eigenvalues of singular pencils, but do not consider corresponding eigenvectors. However, as we will see in Section 4 , the eigenvectors of the perturbed pencil play a key role in our approach.

Instead of eigenvectors and deflating subspaces, the concept of reducing subspaces introduced in [41] is more adequate in the case of singular pencils. We say that a subspace $\mathcal{M}$ is a reducing subspace for the pencil $A-\lambda B$ if $\operatorname{dim}(A \mathcal{M}+B \mathcal{M})=\operatorname{dim}(\mathcal{M})-k$, where $k$ is the number of right singular blocks. In the example above, the reducing subspace associated with the eigenvalue $\lambda_{0}=1$ is exactly given by all vectors $x(\alpha, \beta)$ with $\alpha, \beta \in \mathbb{C}$. The minimal reducing subspace $\mathcal{M}_{\mathrm{RS}}(A, B)$ is the intersection of all reducing subspaces. It is unique and can be numerically computed in a stable way from the generalized upper triangular form (Guptri); see, e.g., [13. Guptri exists for every pencil $A-\lambda B$ and has the form

$$
P^{*}(A-\lambda B) Q=\left[\begin{array}{ccc}
A_{\mathrm{r}}-\lambda B_{\mathrm{r}} & \times & \times \\
0 & A_{\mathrm{reg}}-\lambda B_{\mathrm{reg}} & \times \\
0 & 0 & A_{l}-\lambda B_{l}
\end{array}\right],
$$

where the matrices $P$ and $Q$ are unitary, $A_{\mathrm{r}}-\lambda B_{\mathrm{r}}$ has only right singular blocks in its $\mathrm{KCF}$, $A_{l}-\lambda B_{l}$ has only left singular blocks in its KCF, and $A_{\text {reg }}-\lambda B_{\text {reg }}$ has only regular blocks in its $\mathrm{KCF}$. We will briefly come back to a minimal reducing subspace in Section 3.3 .

3. Motivation and applications. Before proposing our new method, we first review some motivating applications where one is interested in computing the eigenvalues of a singular pencil, besides the computation of transmission zeros already mentioned in the introduction.

3.1. Differential algebraic equations and descriptor systems. Linear differential algebraic equations (DAEs) with constant coefficients have the general form

$$
E \dot{x}=A x+f(t), \quad x\left(t_{0}\right)=x_{0},
$$

where $E, A \in \mathbb{R}^{k, n}, t_{0} \in \mathbb{R}, x_{0} \in \mathbb{R}^{n}$, and $f:\left[t_{0}, \infty\right) \rightarrow \mathbb{R}^{k}$ is a given inhomogeneity; see [23]. Linear time-invariant descriptor systems consist of a DAE combined with a system input and output and take the form

$$
\begin{aligned}
E \dot{x} & =A x+B u, \quad x\left(t_{0}\right)=x_{0}, \\
y & =C x+D u,
\end{aligned}
$$

where, in addition, $B \in \mathbb{R}^{k, m}, C \in \mathbb{R}^{p, n}, D \in \mathbb{R}^{p, m}$. Here, $x:\left[t_{0}, \infty\right) \rightarrow \mathbb{R}^{n}$ stands for the state of the descriptor system, $u:\left[t_{0}, \infty\right) \rightarrow \mathbb{R}^{m}$ is the input and $y:\left[t_{0}, \infty\right) \rightarrow \mathbb{R}^{p}$ the output. As highlighted in [3], the problem may be well-posed even if the underlying pencil $A-\lambda E$ is singular. Indeed, even in the singular case the corresponding DAE may have a solution (even unique) for particular inhomogeneities $f$ and special initial conditions $\left(t_{0}, x_{0}\right)$. 
3.2. Double eigenvalue problem. Given two $n \times n$ matrices $A$ and $B$, we are interested in all values $\lambda$ such that $A+\lambda B$ has a double eigenvalue. For the generic case of a double eigenvalue, we look for independent vectors $x$ and $y$ such that

$$
\begin{aligned}
(A+\lambda B-\mu I) x & =0, \\
(A+\lambda B-\mu I)^{2} y & =0,
\end{aligned}
$$

for some $\mu$. This application is discussed in [33], together with a staircase algorithm to solve it; see also [21]. In the generic case, the problem has $n(n-1)$ solutions.

In [33], the problem is solved by a linearization of the second equation and followed by the solution of the obtained singular two-parameter eigenvalue problem. The wanted values $\lambda$ are eigenvalues of the singular pencil $\Delta_{1}-\lambda \Delta_{0}$ of size $3 n^{2} \times 3 n^{2}$, where

$$
\Delta_{1}=A \otimes R-I \otimes P, \quad \Delta_{0}=B \otimes R-I \otimes Q,
$$

for

$$
P=\left[\begin{array}{ccc}
A^{2} & A B+B A & -2 A \\
0 & I & 0 \\
0 & 0 & I
\end{array}\right], Q=\left[\begin{array}{rrr}
0 & B^{2} & -B \\
-I & 0 & 0 \\
0 & 0 & 0
\end{array}\right], R=\left[\begin{array}{rrr}
0 & -B & I \\
0 & 0 & 0 \\
-I & 0 & 0
\end{array}\right]
$$

Generically, the pencil $\Delta_{1}-\lambda \Delta_{0}$ will have normal rank $3 n^{2}-n$. For more details, see [33] as well as [19] for other possible linearizations.

3.3. Singular two-parameter eigenvalue problems. In the singular two-parameter eigenvalue problem we are given a pair of singular pencils $\Delta_{1}-\lambda \Delta_{0}$ and $\Delta_{2}-\mu \Delta_{0}$ (see also (7.2) and (7.3)), and the goal is to find finite regular eigenvalues $\left(\lambda_{0}, \mu_{0}\right)$, where (see, e.g., [31] for more details):

- $\lambda_{0}$ is a finite eigenvalue of $\Delta_{1}-\lambda \Delta_{0}$,

- $\mu_{0}$ is a finite eigenvalue of $\Delta_{2}-\mu \Delta_{0}$,

- and there exists a nonzero vector $z$ such that $\left(\Delta_{1}-\lambda_{0} \Delta_{0}\right) z=0,\left(\Delta_{2}-\mu_{0} \Delta_{0}\right) z=0$, and $z \notin \mathcal{M}_{\mathrm{RS}}\left(\Delta_{i}, \Delta_{0}\right)$ for $i=1,2$.

This problem requires more than just solving one singular GEP. We discuss it in more details in Section 7 and present a new numerical method for its solution.

4. Rank-completing perturbations of singular pencils. Let $A-\lambda B$ be a singular pencil, where $A, B \in \mathbb{C}^{n, n}$ and $\operatorname{nrank}(A, B)=n-k$. In this section we investigate the effect of rank-completing perturbations, i.e., rank- $k$ generic perturbations of the form

$$
\widetilde{A}-\lambda \widetilde{B}:=A-\lambda B+\tau\left(U D_{A} V^{*}-\lambda U D_{B} V^{*}\right),
$$

where $D_{A}, D_{B} \in \mathbb{C}^{k, k}$ are diagonal matrices such that $D_{A}-\lambda D_{B}$ is a regular pencil, $U, V \in \mathbb{C}^{n, k}$ have full column rank, and $\tau \in \mathbb{C}$ is nonzero. We investigate the dependence of eigenvalues and eigenvectors of the perturbed pencils on $\tau$.

Above and in the following, the term generic is understood in the following sense. A set $\mathcal{A} \subseteq \mathbb{C}^{m}$ is called algebraic if it is the set of common zeros of finitely many complex polynomials in $m$ variables, and $\mathcal{A}$ is called proper if $\mathcal{A} \neq \mathbb{C}^{m}$. A set $\Omega \subseteq \mathbb{C}^{m}$ is called generic if its complement is contained in a proper algebraic set. In this sense we say that a property $\mathcal{P}$ holds generically with respect to the entries of $U$ and $V$, if there exists a generic set $\Omega \subseteq\left(\mathbb{C}^{n, k}\right)^{2}$ (where we interpret $\left(\mathbb{C}^{n, k}\right)^{2}$ as $\left.\mathbb{C}^{2 n k}\right)$ such that $\mathcal{P}$ holds for all pencils of the form (4.1) with $(U, V) \in \Omega$.

REMARK 4.1. Perturbations of the form (4.1) are not the most general perturbations of rank $k$. Indeed, completing $U$ and $V$ to nonsingular matrices $P=\left[\begin{array}{ll}U & \widetilde{U}\end{array}\right]$ and $Q=\left[\begin{array}{ll}V & \widetilde{V}\end{array}\right]$, we obtain 
that

$$
P^{-1}\left(U D_{A} V^{*}-\lambda U D_{B} V^{*}\right)\left(Q^{*}\right)^{-1}=\left[\begin{array}{cc}
D_{A}-\lambda D_{B} & 0 \\
0 & 0
\end{array}\right]
$$

which means that the perturbation pencil $U D_{A} V^{*}-\lambda U D_{B} V^{*}$ has the regular part $D_{A}-\lambda D_{B}$ of size $k \times k$, while, generically, a matrix pencil of nrank $k<n$ would have no regular part [8]. Thus, more generally one could consider perturbations of the form

$$
\left(A+U_{1} V_{1}^{*}+V_{2} U_{2}^{*}, B+U_{1} W_{1}^{*}+W_{2} U_{2}^{*}\right),
$$

where $U_{1}, V_{1}, W_{1} \in \mathbb{C}^{n, \ell}$ and $U_{2}, V_{2}, W_{2} \in \mathbb{C}^{n, k-\ell}$ have full column rank for $\ell \in\{0,1, \ldots, k\}$. However, we will restrict ourselves to perturbations of the form (4.1), because of their favorable properties.

Generically, a rank completing perturbation (4.1) of $A-\lambda B$ will result in a regular perturbed pencil $\widetilde{A}-\lambda \widetilde{B}$. We will show in the following, that generically the $\mathrm{KCF}$ of $\widetilde{A}-\lambda \widetilde{B}$ is for all $\tau \neq 0$ given by

$$
\left[\begin{array}{ccc}
R_{\mathrm{reg}}(\lambda) & 0 & 0 \\
0 & R_{\mathrm{pre}}(\lambda) & 0 \\
0 & 0 & R_{\mathrm{ran}}(\lambda)
\end{array}\right]
$$

where $R_{\text {reg }}(\lambda)$ is the regular part of the original pencil $A-\lambda B, R_{\text {pre }}(\lambda)=D_{A}-\lambda D_{B}$ and $R_{\text {ran }}(\lambda)$ only has simple eigenvalues that are different from the eigenvalues of $R_{\text {reg }}(\lambda)$ and $R_{\text {pre }}(\lambda)$. Thus, the eigenvalues of $\widetilde{A}-\lambda \widetilde{B}$ are exactly the (say) $p$ eigenvalues of the original pencil $A-\lambda B$ (counted with multiplicities) and $n-p$ newly generated eigenvalues which consist of $k$ "prescribed" eigenvalues which are the eigenvalues of the perturbation pencil $U\left(D_{A}-\lambda D_{B}\right) V^{*}$, and $n-p-k$ "random" eigenvalues that are gathered in the part $R_{\text {ran }}(\lambda)$.

We start by showing that under a rank-completing perturbation, the regular part of $A-\lambda B$ will stay invariant in the above sense. Although our main focus are square pencils, we will state some results in more generality covering also the case of rectangular pencils. The following proposition is a generalization of [28, Thm. 4.2] (which deals with Hermitian pencils) to a block case without any specific structure in $A$ and $B$.

Proposition 4.2. Let $A-\lambda B$ be an $n \times m$ singular matrix pencil having at least $k$ left minimal indices, and let $U \in \mathbb{C}^{n, k}$. Then generically (with respect to the entries of $U$ ) there exist nonsingular matrices $P, Q$ such that

$$
P(A-\lambda B) Q=\left[\begin{array}{cc}
R(\lambda) & 0 \\
0 & S(\lambda)
\end{array}\right] \quad \text { and } \quad P U=\left[\begin{array}{c}
0 \\
\widetilde{U}
\end{array}\right],
$$

where $R(\lambda)$ and $S(\lambda)$ are the regular and singular parts of $A-\lambda B$, respectively, and $P U$ is partitioned conformably with $P(A-\lambda B) Q$.

Proof. Without loss of generality we may assume that $A-\lambda B$ is already in the KCF

$$
A-\lambda B=\left[\begin{array}{cc}
R(\lambda) & 0 \\
0 & S(\lambda)
\end{array}\right], \quad R(\lambda)=\left[\begin{array}{cc}
J-\lambda I & 0 \\
0 & I-\lambda N
\end{array}\right],
$$

where $S(\lambda)$ is the singular part and $R(\lambda)$ is the regular part of $A-\lambda B$ with $J, N$ in Jordan normal canonical form and, in addition, $N$ nilpotent. Our main strategy is to use the part of $P$ that corresponds to $k$ arbitrarily chosen left minimal indices to introduce zeros in the components of $U$ that correspond to the regular part of $A-\lambda B$. Here, we can treat the components of $U$ 
corresponding to the "finite eigenvalue part" $J-\lambda I$ and the "infinite eigenvalue part" $I-\lambda N$ separately and we will give the proof only for the first case as the proof for the "infinite eigenvalue part" is completely analogous. Since we will only transform parts of the singular part $S(\lambda)$ that correspond to $k$ arbitrarily chosen left minimal indices and leave all other parts of $S(\lambda)$ unchanged, it is sufficient to assume that $S(\lambda)$ consists of only $k$ singular blocks corresponding to $k$ left minimal indices and that $R(\lambda)$ does not have a part corresponding to infinite eigenvalues. This assumption will simplify the notation considerably.

Thus, we assume that $U$ and $A-\lambda B$ have the forms

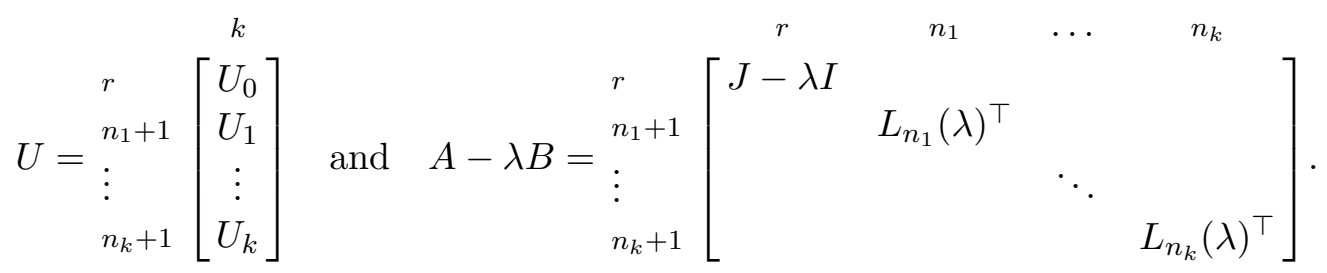

In the following, we will use the notation $G_{\ell}=\left[\begin{array}{ll}0 & I_{\ell}\end{array}\right]^{\top}$ and $H_{\ell}=\left[\begin{array}{ll}I_{\ell} & 0\end{array}\right]^{\top}$ to write $L_{\ell}(\lambda)^{\top}=$ $G_{\ell}-\lambda H_{\ell}$ for a singular block corresponding to a left minimal index $\ell$. For the transformation matrices $P, Q$ we make the ansatz

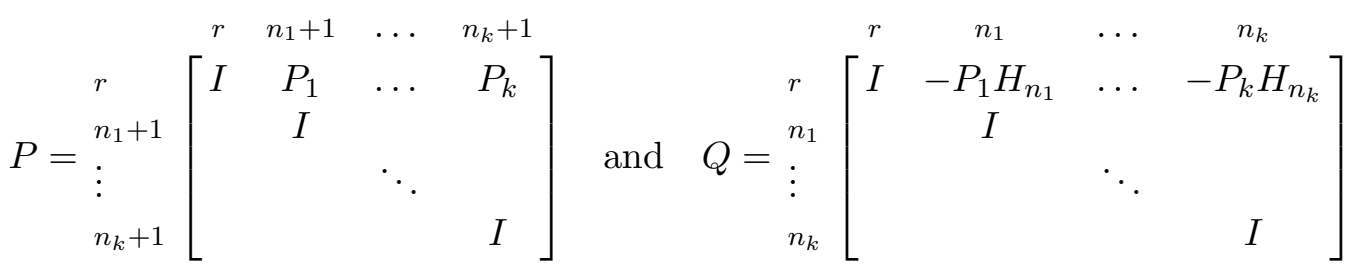

As $P$ and $Q$ are designed in such a way that $B$ remains unchanged, we get

$$
P(A-\lambda B) Q=\left[\begin{array}{cccc}
J-\lambda I & P_{1} G_{n_{1}}-J P_{1} H_{n_{1}} & \ldots & P_{k} G_{n_{k}}-J P_{k} H_{n_{k}} \\
& L_{n_{1}}(\lambda)^{\top} & & \\
& & \ddots & \\
& & & L_{n_{k}}(\lambda)^{\top}
\end{array}\right] .
$$

It remains to find solutions $P_{i}$ to the equations $P_{i} G_{n_{i}}-J P_{i} H_{n_{i}}=0$ for $i=1, \ldots, k$ to obtain $P(A-\lambda B) Q=A-\lambda B$. Setting $P_{i}=\left[\begin{array}{llll}p_{i, 0} & p_{i, 1} & \ldots & p_{i, n_{i}}\end{array}\right]$ for $i=1, \ldots, k$, the equations to be solved take the form

$$
\left[\begin{array}{lll}
p_{i, 1} & \ldots & p_{i, n_{i}}
\end{array}\right]=P_{i} G_{n_{i}}=J P_{i} H_{n_{i}}=\left[\begin{array}{lll}
J p_{i, 0} & \ldots & J p_{i, n_{i}-1}
\end{array}\right], \quad i=1, \ldots, k .
$$

Thus, we may choose $P_{i}=\left[\begin{array}{lllll}p_{i, 0} & J p_{i, 0} & \ldots & J^{n_{i}} p_{i, 0}\end{array}\right]$, where $p_{i, 0} \in \mathbb{C}^{r}$ is arbitrary. We will now use the freedom in the choice of $p_{i, 0}$ to guarantee that $P U$ has the desired form. To this end, let $u_{i, 0}^{\top}, \ldots, u_{i, n_{i}}^{\top} \in \mathbb{C}^{k}$ be the rows of $U_{i}, i=1, \ldots, k$. Then the first block component of $P U$ is given by $U_{0}+P_{1} U_{1}+\cdots+P_{k} U_{k}$ and to make it zero, we have to solve the equation

$$
-U_{0}=\sum_{i=1}^{k} P_{i} U_{i}=\sum_{i=1}^{k} \sum_{j=0}^{n_{i}} J^{j} p_{i, 0} u_{i, j}^{\top}
$$

for $p_{1,0}, \ldots, p_{k, 0}$. Using the vec-operation that "vectorizes" a matrix by stacking the columns on top of each other and recalling the well-known identity $\operatorname{vec}(X Y Z)=\left(Z^{\top} \otimes X\right) \operatorname{vec}(Y)$ for matrices $X, Y, Z$, where $\otimes$ denotes the Kronecker product, we obtain that

$$
-\operatorname{vec}\left(U_{0}\right)=\sum_{i=1}^{k} \sum_{j=0}^{n_{i}}\left(u_{i, j} \otimes J^{j}\right) p_{i, 0}=M \cdot\left[\begin{array}{c}
p_{1,0} \\
\vdots \\
p_{k, 0}
\end{array}\right],
$$


where

$$
M=\left[\sum_{j=0}^{n_{1}} u_{1, j} \otimes J^{j} \ldots \sum_{j=0}^{n_{k}} u_{k, j} \otimes J^{j}\right] \in \mathbb{C}^{r k, r k} .
$$

The determinant of $M$ is a polynomial in the $n k$ entries of $U$ (in fact, it only depends on the entries of $\left.U_{1}, \ldots, U_{k}\right)$ which is nonzero for the particular choice $u_{1,0}=e_{1}, \ldots, u_{k, 0}=e_{k}$ and $u_{i, j}=0$ for $j>0$, where $e_{1}, \ldots, e_{k}$ denote the standard basis vectors of $\mathbb{C}^{k}$. (Indeed, in this case $M$ is just the identity of size $r k \times r k$.) Thus, generically (with respect to the entries of $U$ ), the matrix $M$ is invertible, so equation (4.4) and thus also (4.3) can be uniquely solved for $p_{1,0}, \ldots, p_{k, 0}$ which finishes the proof.

The next result shows that the canonical form of the regular part of the original pencil stays invariant under a generic rank-completing perturbation of the form (4.1). Concerning the eigenvalues of the perturbed pencil that are also eigenvalues of the original singular pencil, the result also states that the corresponding left and right eigenvectors satisfy a particular orthogonality relation.

TheOREM 4.3. Let $A-\lambda B$ be an $n \times n$ singular pencil of normal rank $n-k$, let $U, V \in \mathbb{C}^{n, k}$ have full column rank and let $D_{A}, D_{B} \in \mathbb{C}^{k, k}$ be such that $D_{A}-\lambda D_{B}$ is regular and all eigenvalues of $D_{A}-\lambda D_{B}$ are distinct from the eigenvalues of $A-\lambda B$. Then, generically with respect to the entries of $U$ and $V^{*}$, the following statements hold for the pencil (4.1):

1. For each $\tau \neq 0$, there exist nonsingular matrices $\widetilde{P}$ and $\widetilde{Q}$ such that

$$
\widetilde{P}(\widetilde{A}-\lambda \widetilde{B}) \widetilde{Q}=\left[\begin{array}{cc}
R(\lambda) & 0 \\
0 & R_{\text {new }}(\lambda)
\end{array}\right],
$$

where $R(\lambda)$ is the regular part of the original pencil $A-\lambda B$, and $R_{\text {new }}(\lambda)$ is regular and all its eigenvalues are distinct from the eigenvalues of $R(\lambda)$.

2. If $\lambda_{0}$ is a finite eigenvalue of $A-\lambda B$, i.e., $\operatorname{rank}\left(A-\lambda_{0} B\right)<n-k$, then $\lambda_{0}$ is an eigenvalue of (4.1) for each $\tau \neq 0$. Furthermore, the right null space $\mathcal{N}_{r}\left(\lambda_{0}\right):=\operatorname{ker}\left(\widetilde{A}-\lambda_{0} \widetilde{B}\right)$ and the left null space $\mathcal{N}_{l}\left(\lambda_{0}\right):=\operatorname{ker}\left(\left(\widetilde{A}-\lambda_{0} \widetilde{B}\right)^{*}\right)$ are both constant in $\tau \neq 0$. In addition:

(a) $\mathcal{N}_{r}\left(\lambda_{0}\right) \perp \operatorname{span}(V)$, i.e., if $x$ is a right eigenvector of $\widetilde{A}-\lambda \widetilde{B}$ associated with $\lambda_{0}$, then $V^{*} x=0$.

(b) $\mathcal{N}_{l}\left(\lambda_{0}\right) \perp \operatorname{span}(U)$, i.e., if $y$ is a left eigenvector of $\widetilde{A}-\lambda \widetilde{B}$ associated with $\lambda_{0}$, then $U^{*} y=0$.

3. If $\infty$ is a eigenvalue of $A-\lambda B$, i.e., $\operatorname{rank}(B)<n-k$, then $\infty$ is an eigenvalue of (4.1) for each $\tau \neq 0$. The right and left null spaces $\mathcal{N}_{r}(\infty):=\operatorname{ker}(\widetilde{B})$ and $\mathcal{N}_{l}(\infty):=\operatorname{ker}\left(\widetilde{B}^{*}\right)$ are both constant in $\tau \neq 0$. In addition:

(a) $\mathcal{N}_{r}(\infty) \perp \operatorname{span}(V)$, i.e., if $x$ is a right eigenvector of $\widetilde{A}-\lambda \widetilde{B}$ associated with $\infty$, then $V^{*} x=0$.

(b) $\mathcal{N}_{l}(\infty) \perp \operatorname{span}(U)$, i.e., if $y$ is a left eigenvector of $\widetilde{A}-\lambda \widetilde{B}$ associated with $\infty$, then $U^{*} y=0$.

Proof. First, we will assume that $A-\lambda B$ does not have one of the eigenvalues 0 or $\infty$ and we will show 1) and 2) for this particular case.

Applying Proposition 4.2, there generically exist nonsingular matrices $P, Q$ such that

$$
P(A-\lambda B) Q=\left[\begin{array}{cc}
R(\lambda) & 0 \\
0 & S(\lambda)
\end{array}\right], \quad P U=\left[\begin{array}{c}
0 \\
U_{2}
\end{array}\right], \quad Q^{*} V=\left[\begin{array}{c}
V_{1} \\
V_{2}
\end{array}\right],
$$

where $R(\lambda)$ and $S(\lambda)$ are the regular and singular parts of $A-\lambda B$, respectively, both being in $\mathrm{KCF}$, and where $U$ and $V$ are partitioned conformably with $A-\lambda B$. We will now show 1) and 2): 
1) Since $A-\lambda B$ is square and of normal rank $n-k$, it has exactly $k$ left minimal indices, say $n_{1}, \ldots, n_{k}$ and exactly $k$ right minimal indices, say $m_{1}, \ldots, m_{k}$. We may assume without loss of generality that they are paired up to form square blocks of one left and right minimal index each, i.e., we may assume that $S(\lambda)$ has the block diagonal form

$$
S(\lambda)=\operatorname{diag}\left(\left[\begin{array}{cc}
L_{n_{1}}(\lambda)^{\top} & 0 \\
0 & L_{m_{1}}(\lambda)
\end{array}\right], \ldots,\left[\begin{array}{cc}
L_{n_{k}}(\lambda)^{\top} & 0 \\
0 & L_{m_{k}}(\lambda)
\end{array}\right]\right) .
$$

Then the perturbed pencil takes the form

$$
P(\widetilde{A}-\lambda \widetilde{B}) Q=\left[\begin{array}{cc}
R(\lambda) & 0 \\
\tau U_{2}\left(D_{A}-\lambda D_{B}\right) V_{1}^{*} & R_{\text {new }}(\lambda)
\end{array}\right],
$$

where

$$
R_{\text {new }}(\lambda):=S(\lambda)+\tau U_{2}\left(D_{A}-\lambda D_{B}\right) V_{2}^{*} .
$$

Clearly, the determinant of $P(\widetilde{A}-\lambda \widetilde{B}) Q$ is equal to $\operatorname{det} R(\lambda) \cdot \operatorname{det} R_{\text {new }}(\lambda)$ and from the definition of $R_{\text {new }}(\lambda)$ it is clear that the coefficients of $\operatorname{det} R_{\text {new }}(\lambda)$ are polynomials in the entries of $U_{2}$ and $V_{2}^{*}$ and thus also of $U$ and $V^{*}$.

Now let $\lambda_{0}$ be an eigenvalue of $R(\lambda)$, i.e., $\operatorname{det} R\left(\lambda_{0}\right)=0$. Note that if $e_{j, \ell}$ denotes the $j$ th standard basis vector of $\mathbb{C}^{\ell}$, and $F_{i}=\left(\alpha_{i}-\lambda_{0} \beta_{i}\right) e_{n_{i}+1, n_{i}+m_{i}+1} e_{n_{i}+1, n_{i}+m_{i}+1}^{*}$, then

$$
\operatorname{det}\left(\left[\begin{array}{cc}
L_{n_{i}}\left(\lambda_{0}\right)^{\top} & 0 \\
0 & L_{m_{i}}\left(\lambda_{0}\right)
\end{array}\right]+F_{i}\right)=\left(-\lambda_{0}\right)^{n_{i}}\left(\alpha_{i}-\lambda_{0} \beta_{i}\right) .
$$

Thus, with $e_{j}$ the $j$ th standard basis vector in $\mathbb{C}^{n}$, for the particular choice

$$
U_{2}=V_{2}=\left[\begin{array}{llll}
e_{n_{1}+1} & e_{n_{1}+m_{1}+1+n_{2}+1} & \ldots & e_{n_{1}+m_{1}+1+\cdots+n_{k-1}+m_{k-1}+1+n_{k}+1}
\end{array}\right]
$$

we obtain that

$$
\operatorname{det} R_{\text {new }}\left(\lambda_{0}\right)=\tau^{k}\left(-\lambda_{0}\right)^{n_{1}+\cdots+n_{k}}\left(\alpha_{1}-\lambda_{0} \beta_{1}\right) \cdots\left(\alpha_{k}-\lambda_{0} \beta_{k}\right)
$$

which is nonzero as the eigenvalues of $D_{A}-\lambda D_{B}$ are by hypothesis distinct from $\lambda_{0}$. But then $\operatorname{det} R_{\text {new }}\left(\lambda_{0}\right)$ is generically nonzero (the set of all $\left(U, V^{*}\right)$ for which $\operatorname{det} R_{\text {new }}\left(\lambda_{0}\right)=0$ is by definition an algebraic set, because $\operatorname{det} R_{\text {new }}\left(\lambda_{0}\right)$ is a polynomial in the entries of $U$ and $V^{*}$ ) which shows that $R_{\text {new }}\left(\lambda_{0}\right)$ is generically regular and does not have $\lambda_{0}$ as an eigenvalue. Since intersections of finitely many generic sets are still generic we can conclude that the spectra of $R(\lambda)$ and $R_{\text {new }}(\lambda)$ are disjoint. But then it immediately follows from [25, Lemma 6.11] and [16, XII.2, Thm. 2] that the perturbed pencil $\widetilde{A}-\lambda \widetilde{B}$ has the KCF as given in (4.5).

2) Let $\lambda_{0}$ be a eigenvalue of $A-\lambda B$ and thus of $R(\lambda)$. It then follows directly from 1) that $\lambda_{0}$ is also an eigenvalue of $\widetilde{A}-\lambda \widetilde{B}$ for each $\tau \neq 0$. For the moment, let $\tau \neq 0$ be fixed and let the columns of $Y$ form a basis of the left null space $\mathcal{N}_{l}\left(\lambda_{0}\right)$ of $\widetilde{A}-\lambda \widetilde{B}$. Partition

$$
Y^{*} P^{-1}=\left[\begin{array}{ll}
Y_{1}^{*} & Y_{2}^{*}
\end{array}\right]
$$

conformably with the partition in (4.6). Since $\lambda_{0}$ is not an eigenvalue of $R_{\text {new }}(\lambda)$ we obtain from $Y^{*}\left(\widetilde{A}-\lambda_{0} \widetilde{B}\right)=0$ that $Y_{2}=0$. But this implies that the columns of $Y$ form a basis for the left null space $\mathcal{N}_{l}\left(\lambda_{0}\right)$ for all values $\tau \neq 0$ as the construction of the transformation matrices $P$ and $Q$ only depends on $A, B$, and $U$, but not on $\tau$. Furthermore, we obtain

$$
Y^{*} U=Y^{*} P^{-1} P U=\left[\begin{array}{ll}
Y_{1}^{*} & 0
\end{array}\right] \cdot\left[\begin{array}{c}
0 \\
U_{2}
\end{array}\right]=0,
$$


i.e., $N_{l}\left(\lambda_{0}\right)$ is orthogonal to the space spanned by the columns of $U$.

Observe that the statement on the right null space $\mathcal{N}_{r}\left(\lambda_{0}\right)$ does not follow immediately from the partitioning in (4.6) as in general we have $V_{1} \neq 0$. But we can apply the already proved part of the theorem to the pencil $A^{*}-\lambda B^{*}$ and the perturbation $V\left(D_{A}^{*}-\lambda D_{B}^{*}\right) U^{*}$ to obtain the corresponding statements for the right null space $N_{r}\left(\lambda_{0}\right)$. This finishes the proof of 2).

Finally, assume that $A-\lambda B$ does have one of the eigenvalues 0 or $\infty$. Then apply a Möbius transformation of the form

$$
\mathrm{M}_{\alpha, \beta}(A-\lambda B):=\alpha A+\beta B-\lambda(\alpha B-\beta A)
$$

where $\alpha, \beta \in \mathbb{R}$ are such that $\alpha^{2}+\beta^{2}=1$ and such that $\mathrm{M}_{\alpha, \beta}(A-\lambda B)$ does neither have the eigenvalues 0 nor $\infty$. Note that this Möbius transformation just has the effect of "rotating" eigenvalues on the extended real line $\mathbb{R} \cup\{\infty\}$, but it leaves eigenvectors and the Jordan structure invariant, see, e.g., [26]. The result then follows by applying the already proved parts of the theorem on $\mathrm{M}_{\alpha, \beta}(A-\lambda B)$ followed by applying the inverse Möbius transformation

$$
\mathrm{M}_{\alpha,-\beta}(C-\lambda D):=\alpha C-\beta D-\lambda(\alpha D+\beta C) .
$$

to give the corresponding statements for $A-\lambda B$. In particular, this shows 3 ).

REMARK 4.4. We mention that part 1) in Theorem 4.3 is in line with one of the main results of [7], where it was shown that generically the regular part of a singular pencil stays invariant under generic perturbations that do not make the pencil regular. Part 1) of Theorem 4.3 extends this result (in the sense of the theorem) to the case of rank-completing perturbation. Clearly, the regular part of the pencil will be completely changed if generic perturbations of a rank larger than the difference of the size and the normal rank of the pencil are applied.

Theorem 4.3 characterizes the properties of the eigenvalues from the block $R$ of the perturbed pencil $\widetilde{A}-\lambda \widetilde{B}$ as in (4.1), i.e., of the eigenvalues that coincide with the eigenvalues of the unperturbed pencil. We will next investigate the properties of the eigenvalues from the newly created block $R_{\text {new }}$. We start with the following lemma that will be needed for the main results. The values $\gamma_{1}, \ldots, \gamma_{k}$ in the lemma are the eigenvalues that we will prescribe later in Theorem 4.6 using the matrices $D_{A}$ and $D_{B}$.

Lemma 4.5. Let $A-\lambda B$ be an $n \times n$ singular pencil of normal rank $n-k$ with left minimal indices $n_{1}, \ldots, n_{k}$ and right minimal indices $m_{1}, \ldots, m_{k}$. Furthermore, let $U, V \in \mathbb{C}^{n, k}$ have full column rank, $N:=n_{1}+\cdots+n_{k}, M:=m_{1}+\cdots+m_{k}$, and let $\gamma_{1}, \ldots, \gamma_{k} \in \mathbb{C}$ be given values that are distinct from the eigenvalues of $A-\lambda B$. Then, generically with respect to the entries of $U$ and $V^{*}$, the following statements hold:

1. There exist exactly $M$ pairwise distinct values $\alpha_{1}, \ldots, \alpha_{M}$ different from the eigenvalues of $A-\lambda B$ and different from $\gamma_{1}, \ldots, \gamma_{k}$ such that for each $\alpha_{i}$ there exists a nonzero vector $z_{i}$ with $\left(A-\alpha_{i} B\right) z_{i}=0$ and $V^{*} z_{i}=0$.

2. There exist exactly $N$ pairwise distinct values $\beta_{1}, \ldots, \beta_{N}$ different from the eigenvalues of $A-\lambda B$ and different from $\gamma_{1}, \ldots, \gamma_{k}$ and $\alpha_{1}, \ldots, \alpha_{M}$ such that for each $\beta_{i}$ there exists a nonzero vector $w_{i}$ with $w_{i}^{*}\left(A-\beta_{i} B\right)=0$ and $w_{i}^{*} U=0$.

3. For any given set of $k$ linearly independent vectors $t_{1}, \ldots, t_{k} \in \mathbb{C}^{k}$ there exist nonzero vectors $s_{1}, \ldots, s_{k}$ with $\left(A-\gamma_{i} B\right) s_{i}=0$ and $t_{i}=V^{*} s_{i}$ for $i=1, \ldots, k$.

Proof. 1) Without loss of generality we may assume that $A-\lambda B$ is in KCF such that the blocks $L_{m_{1}}(\lambda), \ldots, L_{m_{k}}(\lambda)$ associated with the right minimal indices appear first in the form. 
Then for each $\alpha \in \mathbb{C}$ different from the eigenvalues of $A-\lambda B$ the columns of

$$
\left[\begin{array}{lll}
q_{1}(\alpha) & \ldots & q_{k}(\alpha)
\end{array}\right]=\left[\begin{array}{ccc}
q_{11}(\alpha) & & 0 \\
& \ddots & \\
0 & & q_{k k}(\alpha) \\
\hline 0 & \ldots & 0
\end{array}\right] \quad \text { with } q_{j j}(\alpha)=\left[\begin{array}{c}
1 \\
\alpha \\
\vdots \\
\alpha^{m_{j}}
\end{array}\right]
$$

form a basis for $\operatorname{ker}(A-\alpha B$ ). (When $\alpha$ is an eigenvalue of $A-\lambda B$, there are additional vectors in $\operatorname{ker}(A-\alpha B)$ since the rank of $A-\alpha B$ drops below the normal rank $n-k$.)

We are looking for $z \neq 0$ and $\alpha$ such that $V^{*} z=0$ and $(A-\alpha B) z=0$. Since we want $\alpha$ to be distinct from the eigenvalues of $A-\lambda B$, the vector $z$ has to be of the form

$$
z=c_{1} q_{1}(\alpha)+\cdots+c_{k} q_{k}(\alpha)
$$

where $c=\left[\begin{array}{lll}c_{1} & \ldots & c_{k}\end{array}\right]^{T} \neq 0$. From $V^{*} z=0$ we get the equation

$$
G(\alpha) c=0
$$

where $G(\alpha)$ is a $k \times k$ matrix whose element $g_{i j}(\alpha)=v_{i}^{*} q_{j}(\alpha)$ is a polynomial in $\alpha$ which generically with respect to the entries of $v_{i}^{*}$ will have degree $m_{j}$ for $i, j=1, \ldots, k$. Equation (4.7) has a nontrivial solution if and only if $\operatorname{det} G(\alpha)=0$, where $\operatorname{det} G(\alpha)$ is a polynomial in $\alpha$ which generically with respect to the entries of $V^{*}$ is of $\operatorname{degree} M$. Thus $\operatorname{det} G(\alpha)$ will have $M$ roots $\alpha_{1}, \ldots, \alpha_{M}$ (counted with multiplicities).

On the other hand, for each fixed $\mu \in \mathbb{C}$, we have that $\operatorname{det} G(\mu)$ is also a polynomial in the entries of $V^{*}=\left[\begin{array}{lll}v_{1} & \ldots & v_{k}\end{array}\right]^{*}$. For the particular choice

$$
v_{1}=e_{1}, \quad v_{2}=e_{m_{1}+2}, \quad \ldots, \quad v_{k}=e_{m_{1}+\cdots+m_{k-1}+k}
$$

we obtain that $v_{i}^{*} q_{j}(\mu)=\delta_{i j}$ so that $G(\mu)=I_{k}$ shows that $\operatorname{det} G(\mu)$ is a nonzero polynomial in the entries of $V^{*}$. It thus follows that generically with respect to the entries of $V^{*}$ we will have $\operatorname{det} G(\mu) \neq 0$, and consequently the fixed value $\mu$ will generically not be among the roots of $G(\alpha)$ as a polynomial in $\alpha$. Since the intersection of finitely many generic sets is still generic, it follows that we can generically exclude finitely many values from the zeros $\alpha_{1}, \ldots, \alpha_{M}$ of $G(\alpha)$. This shows that generically with respect to the entries of $V^{*}$, the values $\alpha_{1}, \ldots, \alpha_{M}$ are different from the eigenvalues of $A-\lambda B$ and also from the given values $\gamma_{1}, \ldots, \gamma_{k}$.

Next we show that the roots $\alpha_{1}, \ldots, \alpha_{M}$ of $p(\alpha):=\operatorname{det} G(\alpha)$ generically are pairwise distinct. This is exactly the case if the discriminant $\operatorname{Disc}(p)$ of $p$ is nonzero. Since $\operatorname{Disc}(p)$ is a polynomial in the entries of $p$ (this is well known, but can also be seen from the fact that the discriminant is a scalar multiple of the determinant of the Sylvester matrix $S\left(p, p^{\prime}\right)$ associated with $p$ and its formal derivative $p^{\prime}$ ), it follows that $\operatorname{Disc}(p)$ is a polynomial with respect to the entries of $V^{*}$. It remains to show that $\operatorname{Disc}(p)$ is a nonzero polynomial (because then we will have that $\operatorname{Disc}(p) \neq 0$ is a generic property with respect to the entries of $\left.V^{*}\right)$, and for this it is enough to show that for a particular choice of the entries of $V$ we have that the values $\alpha_{1}, \ldots, \alpha_{M}$ are pairwise distinct. Now taking $v_{1}=e_{m_{1}+1}-\varepsilon_{1} e_{1}, v_{2}=e_{m_{1}+m_{2}+2}-\varepsilon_{2} e_{m_{1}+2}, \ldots, v_{k}=e_{m_{1}+\cdots+m_{k}+k}-\varepsilon_{k} e_{m_{1}+\cdots+m_{k-1}+k}$, with $\varepsilon_{1}, \ldots, \varepsilon_{k}>0$, we obtain that $v_{i}^{*} q_{j}(\alpha)=\delta_{i j} \alpha^{m_{j}}-\varepsilon_{j}$ and thus $G(\alpha)$ is diagonal and

$$
\operatorname{det} G(\alpha)=\left(\alpha^{m_{1}}-\varepsilon_{1}\right) \cdots\left(\alpha^{m_{k}}-\varepsilon_{k}\right) .
$$

Since the roots of each factor $\left(\alpha^{m_{j}}-\varepsilon_{j}\right)$ are $m_{j}$ pairwise distinct complex numbers lying on a circle centered at zero with radius $\varepsilon_{j}^{1 / m_{j}}$, it remains to choose the values $\varepsilon_{1}, \ldots, \varepsilon_{k}$ in such a way that the $k$ radii are pairwise distinct to obtain the desired example. 
2) In a way similar to the one in 1) we can consider the left null space for $A-\alpha B$ and show the existence of $\beta_{1}, \ldots, \beta_{N}$ and the corresponding nonzero vectors $w_{1}, \ldots, w_{N}$, where now the statements are generic with respect to the entries of $U$. In particular, by interpreting $V$ as already fixed, this shows that generically with respect to the entries of $U$, the values $\beta_{1}, \ldots, \beta_{N}$ are not only different from the eigenvalues of $A-\lambda B$ and $\gamma_{1}, \ldots, \gamma_{k}$, but also from the values $\alpha_{1}, \ldots, \alpha_{M}$ constructed in 1).

3) With the same notation as in 1) we now aim to solve the equations

$$
s_{i}=c_{1} q_{1}\left(\gamma_{i}\right)+\cdots+c_{k} q_{k}\left(\gamma_{i}\right) \text { and } V^{*} s_{i}=t_{i},
$$

or, equivalently, $G\left(\gamma_{i}\right) c=t_{i}$ for $i=1, \ldots, k$. Since $\gamma_{i}$ is different from the values $\alpha_{1}, \ldots, \alpha_{M}$, we have $\operatorname{det} G\left(\gamma_{i}\right) \neq 0$ and hence $G\left(\gamma_{i}\right) c=t_{i}$ is uniquely solvable for $c$ for $i=1, \ldots, k$.

The following theorem encapsulates the main result on the new eigenvalues of our perturbed pencil.

THEOREM 4.6. Let $A-\lambda B$ be an $n \times n$ singular pencil of normal rank $n-k$ with left minimal indices $n_{1}, \ldots, n_{k}$ and right minimal indices $m_{1}, \ldots, m_{k}$. Furthermore, let $U, V \in \mathbb{C}^{n, k}$ have full column rank and let $D_{A}=\operatorname{diag}\left(a_{1}, \ldots, a_{k}\right), D_{B}=\operatorname{diag}\left(b_{1}, \ldots, b_{k}\right) \in \mathbb{C}^{k, k}$ be such that $D_{A}-\lambda D_{B}$ is regular and such that all (not necessarily pairwise distinct) values $\gamma_{i}:=\frac{a_{i}}{b_{i}}, i=1, \ldots, k$, are different from the eigenvalues of $A-\lambda B$. (Here, $\frac{a_{i}}{b_{i}}$ is interpreted as the infinite eigenvalue, if $b_{i}=0$.) Finally, let $N:=n_{1}+\cdots+n_{k}$ and $M:=m_{1}+\cdots+m_{k}$. Then generically with respect to the entries of $U$ and $V^{*}$, the following statements hold:

1. The pencil (4.1) has $M$ simple eigenvalues $\alpha_{1}, \ldots, \alpha_{M}$ which are independent of $\tau \neq 0$, so that for each of these eigenvalues its right eigenvector $x_{i}$ is constant in $\tau \neq 0$ (up to scaling) and satisfies $V^{*} x_{i}=0$, while the left eigenvector $y_{i}$ is a linear function of $\tau$ (up to scaling) and satisfies $U^{*} y_{i} \neq 0$ for all $\tau \neq 0$.

2. The pencil (4.1) has $N$ simple eigenvalues $\beta_{1}, \ldots, \beta_{N}$ which are independent of $\tau \neq 0$, so that for each of these eigenvalues its left eigenvector $y_{i}$ is constant for $\tau \neq 0$ (up to scaling) and satisfies $U^{*} y_{i}=0$, while the right eigenvector $x_{i}$ is a linear function of $\tau$ (up to scaling) and satisfies $V^{*} x_{i} \neq 0$ for all $\tau \neq 0$.

3. For each $\tau \neq 0$ each $\gamma_{i}$ is an eigenvalue of (4.1) with the same algebraic multiplicity as for the pencil $D_{A}-\lambda D_{B}$. Furthermore, the left and right null spaces $\mathcal{N}_{l}\left(\gamma_{i}\right)$ and $\mathcal{N}_{r}\left(\gamma_{i}\right)$ of (4.1) associated with $\gamma_{i}$ are constant in $\tau$. In addition, we have:

(a) $\mathcal{N}_{r}\left(\gamma_{i}\right) \cap \operatorname{ker}\left(V^{*}\right)=\{0\}$, i.e., for each right eigenvector $x$ of (4.1) associated with $\gamma_{i}$ we have $V^{*} x \neq 0$.

(b) $\mathcal{N}_{l}\left(\gamma_{i}\right) \cap \operatorname{ker}\left(U^{*}\right)=\{0\}$, i.e., for each left eigenvector $y$ of (4.1) associated with $\gamma_{i}$ we have $U^{*} y \neq 0$.

(Note that the simplicity of the eigenvalues $\alpha_{1}, \ldots, \alpha_{M}, \beta_{1}, \ldots, \beta_{N}$ implies that they are all different from the eigenvalues of $A-\lambda B$ and $\left.\gamma_{1}, \ldots, \gamma_{k}.\right)$

Proof. Without loss of generality, we may assume that the infinite eigenvalue is not among the eigenvalues of $D_{A}-\lambda D_{B}$. Otherwise, we may as in the proof of Theorem 4.3 apply a Möbius transformation to both $A-\lambda B$ and $D_{A}-\lambda D_{B}$ such that $D_{A}-\lambda D_{B}$ does not have the eigenvalue $\infty$, apply the statement that was proved for this special situation, and finally transform back with the inverse Möbius transformation to obtain the desired result.

Observe that generically with respect to the entries of $U$ and $V^{*}$, the statements of Lemma 4.5 hold, if we take the eigenvalues of the pencil $D_{A}-\lambda D_{B}$ for the values $\gamma_{1}, \ldots, \gamma_{k}$ and take standard basis vectors $e_{1}, \ldots, e_{k}$ from $\mathbb{C}^{k}$ for the vectors $t_{1}, \ldots, t_{k}$. We now show 1)-3).

1) By Lemma 4.5 there exist exactly $M$ pairwise distinct values $\alpha_{1}, \ldots, \alpha_{M}$ different from the eigenvalues of $A-\lambda B$ and from $\gamma_{1}, \ldots, \gamma_{k}$, and nonzero vectors $z_{1}, \ldots, z_{M}$ such that $\left(A-\alpha_{i} B\right) z_{i}=$ 0 and $V^{*} z_{i}=0$ for $i=1, \ldots, M$. From this we obtain that $\left(A-\alpha_{i} B+\tau U D_{A} V^{*}-\alpha_{i} \tau U D_{B} V^{*}\right) z_{i}=$ 
0 which means that $\alpha_{i}$ is an eigenvalue of (4.1) for $\tau \neq 0$ with a right eigenvector $z_{i}$ that is invariant under $\tau$.

Considering now $\tau$ as a variable, it follows that the pencil

$$
G_{i}+\tau H_{i}:=\left(A-\alpha_{i} B\right)+\tau U\left(D_{A}-\alpha_{i} D_{B}\right) V^{*}
$$

is singular. Suppose that $\operatorname{nrank}\left(G_{i}, H_{i}\right)=n-j$ for $j \geq 1$, which means that (4.8) has $j$ right and $j$ left minimal indices. We know from $G_{i} z_{i}=0$ and $H_{i} z_{i}=0$ that one right minimal index is equal to zero. The remaining $j-1$ right minimal indices are all larger than zero, because otherwise there would exist $y_{i} \in \operatorname{ker}\left(G_{i}\right) \cap \operatorname{ker}\left(H_{i}\right)$ linearly independent of $z_{i}$ which implies that $\alpha_{i}$ would be a multiple eigenvalue of (4.1) in contradiction to Lemma 4.5.

Now suppose that (4.8) has a left minimal index being zero. Then there exists a vector $w_{i} \neq 0$ such that $w_{i}^{*} G_{i}=0$ and $w_{i}^{*} H_{i}=0$, which implies $w_{i}^{*} U=0$, because $V$ has full rank and $D_{A}-\alpha_{i} D_{B}$ is nonsingular since $\alpha_{i}$ is different from the eigenvalues of $D_{A}-\lambda D_{B}$. But then by Lemma $4.5 \alpha_{i}$ is equal to one of the values $\beta_{1}, \ldots, \beta_{N}$ which is a contradiction. Thus, all left minimal indices of (4.8) are larger than or equal to one.

Furthermore, we know that $\operatorname{rank}\left(G_{i}\right)=n-k$ and $\operatorname{rank}\left(H_{i}\right)=k$ since $\alpha_{i}$ differs from all finite eigenvalues of $A-\lambda B$ and all eigenvalues of $D_{A}-\lambda D_{B}$. It follows that in the KCF of the pencil (4.8) there are at least $n-k-j$ blocks associated with the eigenvalue infinity and at least $k-j$ blocks associated with the eigenvalue zero.

By a simple computation we obtain that the dimension of the KCF of (4.8) is at least $(n+j-$ $1) \times(n+j-1)$; therefore the only option is $j=1$ and hence (4.8) has exactly one right minimal index (being zero) and exactly one left minimal index, say $p$. Then another simple computation shows that the dimension of the KCF of (4.8) is at least $(n+p-1) \times(n+p-1)$ showing that the left minimal index $p$ must be equal to one. Consequently, there exist linearly independent vectors $w_{i}$ and $z_{i}$ such that

$$
\begin{aligned}
w_{i}^{*}\left(A-\alpha_{i} B\right) & =0, \\
z_{i}^{*}\left(A-\alpha_{i} B\right)+w_{i}^{*} U\left(D_{A}-\alpha_{i} D_{B}\right) V^{*} & =0, \\
z_{i}^{*} U\left(D_{A}-\alpha_{i} D_{B}\right) V^{*} & =0
\end{aligned}
$$

and $w_{i}^{*} U \neq 0$. Up to scaling, the left eigenvector $y_{i}$ of (4.1) associated with $\alpha_{i}$ then has the form $y_{i}(\tau)=w_{i}+\tau z_{i}$ and is a linear function of $\tau$.

2) This follows completely analogously to 1 ).

3) Clearly, the standard basis vectors $e_{1}, \ldots, e_{k}$ are eigenvectors of the pencil $D_{A}-\lambda D_{B}$ associated with the eigenvalues $\gamma_{1}, \ldots, \gamma_{k}$. By Lemma 4.5, there exist $k$ (necessarily linearly independent) vectors $s_{1}, \ldots, s_{k} \in \mathbb{C}^{n}$ such that $\left(A-\gamma_{i} B\right) s_{i}=0$ and $e_{i}=V^{*} s_{i}$ for $i=1, \ldots, k$. Then we have

$$
\left(\widetilde{A}-\gamma_{i} \widetilde{B}\right) s_{i}=\left(A-\gamma_{i} B\right) s_{i}+\tau U\left(D_{A}-\gamma_{i} D_{B}\right) V^{*} s_{i}=0
$$

for each $\tau \neq 0$ for $i=1, \ldots, k$. This implies that the values $\gamma_{1}, \ldots, \gamma_{k}$ are eigenvalues of $\widetilde{A}-\lambda \widetilde{B}$ with the same algebraic multiplicities as for $D_{A}-\lambda D_{B}$. Furthermore, it follows that the null space $\mathcal{N}_{r}\left(\gamma_{i}\right)$ does not depend on $\tau$ and by construction we have $\mathcal{N}_{r}\left(\gamma_{i}\right) \cap \operatorname{ker}\left(V^{*}\right)=\{0\}$.

By applying Lemma 4.5 to the pencil $A^{*}-\lambda B^{*}$ we obtain the analogous statements for the left null spaces $\mathcal{N}_{l}\left(\gamma_{i}\right)$.

Summary 4.7. Summarizing the results from Theorem 4.3 and Theorem 4.6 , let $A-\lambda B$ be an $n \times n$ singular pencil of normal rank $n-k$ with left minimal indices $n_{1}, \ldots, n_{k}$ and right minimal indices $m_{1}, \ldots, m_{k}$, and let $U, V, D_{A}, D_{B}, N$, and $M$ be as in Theorem 4.6. Since the 
regular part of $A-\lambda B$ then has size $r:=n-N-M-k$ and we have found $N+M+k$ new eigenvalues in Theorem 4.6, we have classified all eigenvalues of the perturbed pencil

$$
\widetilde{A}-\lambda \widetilde{B}:=A-\lambda B+\tau\left(U D_{A} V^{*}-\lambda U D_{B} V^{*}\right)
$$

into the following three groups:

1. True eigenvalues: There are $r$ such eigenvalues that are exactly the eigenvalues of $A-\lambda B$. The corresponding right eigenvectors $x$ and left eigenvectors $y$ satisfy $V^{*} x=0$ and $U^{*} y=$ 0 .

2. Prescribed eigenvalues: There are $k$ such eigenvalues that coincide with the $k$ eigenvalues of $D_{A}-\lambda D_{B}$. The corresponding right eigenvectors $x$ and left eigenvectors $y$ satisfy both $V^{*} x \neq 0$ and $U^{*} y \neq 0$.

3. Random eigenvalues: These are the remaining $N+M$ eigenvalues. They are simple and if $\mu$ is such an eigenvalue with the corresponding right eigenvector $x$ and left eigenvector $y$, then we either have $V^{*} x=0$ and $U^{*} y \neq 0$, or $V^{*} x \neq 0$ and $U^{*} y=0$.

Thus, the eigenvalues of $A-\lambda B$ can be identified from the eigenvalues of $\widetilde{A}-\lambda \widetilde{B}$ by investigating orthogonality properties of the corresponding left and right eigenvectors. We will use this observation in the following section for the development of an algorithm for computing the eigenvalues of a singular square pencil.

REMARK 4.8. If $A$ and $B$ are symmetric, then it seems that for our current approach we have to use nonsymmetric rank completing perturbations. Namely, when a symmetric perturbation of the form $\tau U\left(D_{A}-\lambda D_{B}\right) U^{*}$ is used, there is an issue with the third group in Summary 4.7 as random eigenvalues appear either as double real eigenvalues or in complex conjugate pairs, and in the former case the orthogonality constraints cannot be satisfied. We leave the study of structured singular pencils for future research.

5. A perturbation method for singular generalized eigenvalue problems. In this section we explain how in the generic case we can extract the finite true eigenvalues numerically even in double precision by solving only one perturbed eigenvalue problem. The key is formed by the existent or non-existent orthogonality properties of the left and right eigenvectors associated with true, prescribed, and random eigenvalues, respectively.

Let $A-\lambda B$ be a singular $n \times n$ pencil with normal rank $n-k$, where $k>0$. We determine $\operatorname{nrank}(A, B)$ by computing $\operatorname{rank}(A-\zeta B)$ for a random $\zeta$. As we have shown in the previous section, if we take two random $n \times k$ matrices $U$ and $V$ with orthonormal columns, a regular $k \times k$ diagonal pencil $D_{A}-\lambda D_{B}$, and $\tau \neq 0$, then the perturbed pencil (4.1) is regular. The "true" eigenvalues of $A-\lambda B$ (theoretically) remain constant under this perturbation. In contrast, eigenvalues that originate from the singular part of the pencil (the "random" eigenvalues) may be "anywhere in the complex plane". In addition, (4.1) also has $k$ "prescribed" eigenvalues that coincide with the eigenvalues of $D_{A}-\lambda D_{B}$.

In theory, if we compute all eigenvalues $\lambda_{i}$ together with the left and right eigenvectors $x_{i}$ and $y_{i}$ for $i=1, \ldots, n$ of (4.1), then $\max \left(\left\|V^{*} x_{i}\right\|,\left\|U^{*} y_{i}\right\|\right)=0$ for a true eigenvalue and $\max \left(\left\|V^{*} x_{i}\right\|,\left\|U^{*} y_{i}\right\|\right)>0$ for a prescribed or a random eigenvalue, so we can use this criterion to extract the true eigenvalues. In the following we will discuss how the above criterion is affected by computations in finite precision and how it does depend on $\tau$. We will also introduce other criteria that may be used for the same purpose or to further separate true eigenvalues into finite and infinite ones.

If $x_{i}$ and $y_{i}$ are normalized left and right eigenvectors of the perturbed problem (4.1) for an eigenvalue $\lambda_{i}$, we can compute the number

$$
s\left(\lambda_{i}\right)=y_{i}^{*} \widetilde{B} x_{i}=y_{i}^{*} B x_{i}+\tau y_{i}^{*} U D_{B} V^{*} x_{i} .
$$


It is easy to see that $s\left(\lambda_{i}\right) \neq 0$ for a simple finite eigenvalue $\lambda_{i}$. As explained in the following lemma, which is a straightforward generalization of the standard result for a pencil $A-\lambda I$, see, e.g., [42, Sec. 2.9], $1 /\left|s\left(\lambda_{i}\right)\right|$ occurs in the expression for a standard condition number of a simple finite eigenvalue.

LEMMA 5.1. Let $\lambda_{i}$ be a simple finite eigenvalue of a regular matrix pencil $\widetilde{A}-\lambda \widetilde{B}$ and let $x_{i}$ and $y_{i}$ be its normalized left and right eigenvectors. If we perturb the pencil into $(\widetilde{A}+\theta E)-$ $\lambda(\widetilde{B}+\theta F)$ for a small $\theta>0$, then $\lambda_{i}$ perturbs into

$$
\lambda_{i}+\theta \frac{y_{i}^{*} E x_{i}-\lambda_{i} y_{i}^{*} F x_{i}}{s\left(\lambda_{i}\right)}+\mathcal{O}\left(\theta^{2}\right)
$$

If $\lambda_{i}$ is a simple finite true eigenvalue, then $V^{*} x_{i}=0$ and $U^{*} y_{i}=0$, which implies that $s\left(\lambda_{i}\right)=y_{i}^{*} B x_{i}$ does not change with $\tau \neq 0$. For a regular infinite eigenvalue we have $y_{i}^{*} B=0$, $B x_{i}=0, V^{*} x_{i}=0$, and $U^{*} y_{i}=0$, therefore $s(\infty)=0$, again independent of $\tau \neq 0$. On the other hand, we can show that values $s(\lambda)$ of prescribed and random eigenvalues depend on $\tau$ and go to 0 as $\tau$ goes to 0 . For this, we need the following lemma.

Lemma 5.2. Let $A-\lambda B$ be a singular pencil and let $\alpha$ be different from all eigenvalues of $A-\lambda B$, i.e., $\operatorname{rank}(A-\alpha B)=\operatorname{nrank}(A, B)$. If $y^{*}(A-\alpha B)=0$ and $(A-\alpha B) x=0$ then $y^{*} A x=y^{*} B x=0$.

Proof. We know from the structure of the left and right singular blocks that $x \in \mathcal{N}_{r}(\alpha)$ can be written as $x=q_{1}+\alpha q_{2}+\cdots+\alpha^{p} q_{p+1}$, where the vectors $q_{1}, \ldots, q_{p+1}$ form the chain

$$
A q_{1}=0, A q_{2}=B q_{1}, \ldots, A q_{p+1}=B q_{p}, B q_{p+1}=0
$$

for certain $p \geq 0$. Similarly, $y \in \mathcal{N}_{l}(\alpha)$ can be written as $y=w_{1}+\bar{\alpha} w_{2}+\cdots+\bar{\alpha}^{r} w_{r+1}$, where the vectors $w_{1}, \ldots, w_{r+1}$ form the chain

$$
A^{*} w_{1}=0, A^{*} w_{2}=B^{*} w_{1}, \ldots, A^{*} w_{r+1}=B^{*} w_{r}, B^{*} w_{r+1}=0
$$

for certain $r \geq 0$. To show $y^{*} B x=0$ it is enough to show that $w_{i}^{*} B q_{j}=0$ for all $i=1, \ldots, r+1$ and $j=1, \ldots, p+1$. For $i=1$ or $j=p+1$ this follows from $w_{1}^{*} A=0$ and $B q_{p+1}=0$, so we can assume that $i \geq 2$ and $j \leq p$. It follows that $w_{i}^{*} B q_{j}=w_{i}^{*} A q_{j+1}=w_{i-1}^{*} B q_{j+1}$. As we continue in this manner, we eventually reach either $w_{1}^{*} A=0$ or $B q_{p+1}=0$. It follows that $y^{*} B x=0$ and from $y^{*} A x=\alpha y^{*} B x$ we get $y^{*} A x=0$ as well.

LEMma 5.3. Let $\lambda_{i}$ be a prescribed or random eigenvalue of (4.1) under the assumptions of Theorem 4.6, where we assume in addition that all prescribed eigenvalues are algebraically simple. Then there exists a positive constant $c_{i}$ such that $\left|s\left(\lambda_{i}\right)\right|=c_{i}|\tau|$.

Proof. First, let $\lambda_{i}$ be a prescribed eigenvalue. Then by the proof of Theorem 4.6 the corresponding left and right eigenvectors satisfy $y_{i}^{*}\left(A-\lambda_{i} B\right)=0$ and $\left(A-\lambda_{i} B\right) x_{i}=0$ which by Lemma 5.2 implies $y_{i}^{*} B x_{i}=0$. But then we have $\left|s\left(\lambda_{i}\right)\right|=c_{i}|\tau|$ with $c_{i}=\left|y_{i}^{*} U D_{B} V^{*} x_{i}\right|$ and $c_{i}$ must be nonzero, because $\lambda_{i}$ is a simple eigenvalue of $\widetilde{A}-\lambda \widetilde{B}$ for $\tau \neq 0$.

Next, let $\lambda_{i}$ be a random eigenvalue, such that $V^{*} x_{i}=0$ and $U^{*} y_{i} \neq 0$. We know (see the proof of Theorem 4.6) that $y_{i}$ is a linear function of $\tau$ as $y_{i}(\tau)=w_{i}+\tau z_{i}$, where $w_{i}^{*}\left(A-\lambda_{i} B\right)=0$. Since $\left(A-\lambda_{i} B\right) x_{i}=0$, it follows from Lemma 5.2 that $w_{i}^{*} B x_{i}=0$ and $y_{i}^{*} \widetilde{B} x_{i}=y_{i}^{*} B x_{i}=\bar{\tau} z_{i}^{*} B x_{i}$. The case $V^{*} x_{i} \neq 0$ and $U^{*} y_{i}=0$ can be shown analogously.

So, if we take a $\tau$ of small absolute value and if all finite true eigenvalues are simple and none of them is too ill-conditioned, then we can separate the finite true eigenvalues from the remaining ones using the values $s(\lambda)$.

Let $\varepsilon$ be the machine precision and let the matrices $A$ and $B$ be scaled in such way that $\|A\|=\|B\|=1$. If all finite true eigenvalues are simple and not too ill-conditioned, then we 
expect to observe the situation in Table 5.1, where $c>0$ is a constant, independent of $\tau$, and possibly different for each eigenvalue and each entry in the table.

TABLE 5.1

Characteristics of the eigenvalues of the perturbed pencil as in (4.1).

\begin{tabular}{l|l|l|l}
\hline Eigenvalue $\lambda$ & $|s(\lambda)|$ & $\left\|V^{*} x\right\|$ & $\left\|U^{*} y\right\|$ \\
\hline Finite true eigenvalue of $A-\lambda B$ & $c$ & $<c \varepsilon /|\tau|$ & $<c \varepsilon /|\tau|$ \\
Infinite true eigenvalue of $A-\lambda B$ & $<c \varepsilon$ & $<c \varepsilon /|\tau|$ & $<c \varepsilon /|\tau|$ \\
Prescribed eigenvalue of $D_{A}-\lambda D_{B}$ & $c|\tau|$ & $c$ & $c$ \\
Random eigenvalue from an $L_{p}$ block & $c|\tau|$ & $<c \varepsilon /|\tau|$ & $c$ \\
Random eigenvalue from an $L_{p}^{T}$ block & $c|\tau|$ & $c$ & $<c \varepsilon /|\tau|$ \\
\hline
\end{tabular}

We now explain the values in Table 5.1. We will start with column $|s(\lambda)|$ and a finite true eigenvalue, where we assume that all finite true eigenvalues are simple and well-conditioned. It follows that $\lambda$ is a simple eigenvalue of $\widetilde{A}-\lambda \widetilde{B}$, therefore $y^{*} \widetilde{B} x \neq 0$ and, since this value is independent of $\tau$ and $\varepsilon$, we have $|s(\lambda)|=c$. For an infinite eigenvalue we should have $y^{*} \widetilde{B} x=0$ in exact computation, instead, in finite precision, we get $\left|y^{*} \widetilde{B} x\right|<c \varepsilon$. Finally, in the generic case, if $\lambda_{i}$ is a prescribed or random eigenvalue then Lemma 5.3 yields that $\left|s\left(\lambda_{i}\right)\right|=c_{i}|\tau|$ for a positive constant $c_{i}$.

Finally, the values in the columns $\left\|V^{*} x\right\|$ and $\left\|U^{*} x\right\|$ that are marked by $<c \varepsilon /|\tau|$ should be zero in exact arithmetic. In finite precision however, due to the supposed backward stability of the applied eigenproblem solver, the computed eigenvalues and eigenvectors of $\widetilde{A}-\lambda \widetilde{B}$ are exact eigenpairs of a perturbed pencil $\widetilde{A}+E-\lambda(\widetilde{B}+F)$, where $\|E\| \leq \widetilde{c}_{1}\|\widetilde{A}\| \varepsilon$ and $\|F\| \leq \widetilde{c}_{2}\|\widetilde{B}\| \varepsilon$. If we assume that all finite eigenvalues of $\widetilde{A}-\lambda \widetilde{B}$ are simple, then we have the following result on the first-order eigenvector perturbations. The proof is omitted since it is a straightforward generalization of the result for the pencil $A-\lambda I$ from [42, Sec. 2.10].

LEMMA 5.4. Let all finite eigenvalues $\lambda_{i}$ of $\widetilde{A}-\lambda \widetilde{B}$ be simple and let $x_{i}$ and $y_{i}$ be corresponding left and right normalized eigenvectors. If the pencil is perturbed into $\widetilde{A}+\theta E-\lambda(\widetilde{B}+\theta F)$, then the eigenvector $x_{i}$ perturbs into

$$
\widetilde{x}_{i}=x_{i}+\theta \sum_{k=1, k \neq i}^{n} \frac{y_{k}^{*}\left(E-\lambda_{i} F\right) x_{i}}{\left(\lambda_{i}-\lambda_{k}\right) s\left(\lambda_{k}\right)} x_{k}+\mathcal{O}\left(\theta^{2}\right) .
$$

Let $\lambda_{i}$ be a finite true eigenvalue of $A-\lambda B$. Then $\lambda_{i}$ is also an eigenvalue of $\widetilde{A}-\lambda \widetilde{B}$ and $V^{*} x_{i}=0$, where $x_{i}$ is an exact normalized right eigenvector. In finite precision, $x_{i}$ becomes perturbed in the directions of other eigenvectors and by Lemma 5.4 a contribution in the direction of another eigenvector depends on the condition number of the corresponding eigenvalue. The only contributions that affect the value of $\left\|V^{*} \widetilde{x}_{i}\right\|$ are those related to prescribed eigenvalues or random eigenvalues from left singular blocks, as right eigenvectors of other eigenvalues are orthogonal to $V$. As condition numbers of these eigenvalues are equal to $1 /(c|\tau|)$ and $\left\|V^{*} x_{j}\right\|=c$ for the corresponding right eigenvectors, it follows from Lemma 5.4 and the backward stability of the computed eigenpairs that $\left\|V^{*} \widetilde{x}_{i}\right\|<c \varepsilon /|\tau|$.

Next, we discuss appropriate choices for the value $\tau$. If $|\tau|$ is close to $\varepsilon$, then the prescribed and random eigenvalues are very ill-conditioned, and perturbations of eigenvectors may move the values of $\left\|V^{*} x_{i}\right\|$ and $\left\|U^{*} y_{i}\right\|$ far away from zero when they should be close to zero. Therefore, if $|\tau|$ is too small, we may not be able to use the values of $\left\|V^{*} x_{i}\right\|$ and $\left\|U^{*} y_{i}\right\|$ to extract the true eigenvalues. Still, if all finite true eigenvalues are simple, then we may use the values $\left|s\left(\lambda_{i}\right)\right|$ to extract the finite true eigenvalues. 
On the other hand, if $|\tau|$ is large, then all eigenvalues, except the infinite ones, are expected to be well-conditioned which means that the eigenvectors will not change much and the computed left and right eigenvectors will be orthogonal to $V$ or $U$ in finite precision, when they should be. Therefore, for large $|\tau|$, we can first use $\max \left(\left\|V^{*} x_{i}\right\|,\left\|U^{*} y_{i}\right\|\right)$ to extract the true eigenvalues and then use $\left|s\left(\lambda_{i}\right)\right|$ to distinguish the finite true eigenvalues from the infinite one. In practice, we see this as a better option, because it does not depend on finite true eigenvalues being simple. However, we should not choose $|\tau|$ too large as this may decrease the precision of the computed finite true eigenvalues. Since the computed eigenvalues are, due to assumed backward stability, exact eigenvalues of a slightly perturbed pencil $\widetilde{A}-\lambda \widetilde{B}$, it is safe to use $|\tau|$ up to $\|\widetilde{A}\| \approx\|A\|$ and $\|\widetilde{B}\| \approx\|B\|$. Also, since from our analysis it follows that only the absolute value of $\tau$ seems to matter, we suggest to choose $\tau$ real and positive.

Based on the above discussion, we summarize our method in Algorithm 1. Note that we scale the matrices in such way that $\|A\|_{1}=\|B\|_{1}=1$, mainly for convenience, to determine an appropriate default value for $\tau$.

Algorithm 1: Computing finite eigenvalues of a singular pencil $(A, B)$ by a rankcompleting perturbation.

Input: $A$ and $B$, perturbation constant $\tau$ (default $10^{-2}$ ), thresholds $\delta_{1}$ (default $\varepsilon^{1 / 2}$ ) and $\delta_{2}$ (default $10^{2} \varepsilon$ ).

Output: Eigenvalues of the finite regular part.

1: Scale $A=(1 / \alpha) A$ and $B=(1 / \beta) B$, where $\alpha=\|A\|_{1}$ and $\beta=\|B\|_{1}$.

2: $\quad$ Compute $\operatorname{nrank}(A, B): k=\operatorname{rank}(A-\zeta B)$ for $\operatorname{random} \zeta$.

3: Select random $n \times k$ matrices $U$ and $V$ with orthonormal columns.

4: $\quad$ Select diagonal $k \times k$ matrices $D_{A}$ and $D_{B}$ such that the eigenvalues of $\left(D_{A}, D_{B}\right)$ are (likely) different from those of $(A, B)$

(default: choose diagonal elements of $D_{A}$ and $D_{B}$ uniformly random

from the interval $[1,2])$.

5: Compute the eigenvalues $\lambda_{i}, i=1, \ldots, n$, and right and left

eigenvectors $x_{i}$ and $y_{i}$ of $(\widetilde{A}, \widetilde{B})=\left(A+\tau U D_{A} V^{*}, B+\tau U D_{B} V^{*}\right)$.

6: Compute $s_{i}=y_{i}^{*} \widetilde{B} x_{i}$ for $i=1, \ldots, n$.

7: $\quad$ Compute $\zeta_{i}=\max \left(\left\|V^{*} x_{i}\right\|,\left\|U^{*} y_{i}\right\|\right)$ for $i=1, \ldots, n$.

8: $\quad$ Return all eigenvalues $(\alpha / \beta) \lambda_{i}, i=1, \ldots, n$, where $\zeta_{i}<\delta_{1}$ and $\left|s_{i}\right|>\delta_{2}$.

As we will show by experiments in the next section, the above approach seems to work very well in double precision for small or moderate singular pencils. Of course, if some of the eigenvalues are very ill-conditioned (for instance when some of the eigenvalues are multiple), then the method may fail in extracting some of the finite true eigenvalues. However, its advantage over staircase-based methods may be the following observation: if we make a wrong rank decision in a staircase algorithm, then the method usually fails completely and returns no eigenvalues at all; see Example 6.4 in the next section. In contrast, the method proposed here is able to detect, if not all, then at least the well-conditioned finite eigenvalues of the pencil under consideration.

6. Numerical examples. In this section we demonstrate the method with several numerical examples computed in Matlab 2015b. All numerical examples and implementations of the 
algorithms are available in [35.

Example 6.1 . We take a $7 \times 7$ pencil $A-\lambda B$, where

$$
A=\left[\begin{array}{rrrrrrr}
-1 & -1 & -1 & -1 & -1 & -1 & -1 \\
1 & 0 & 0 & 0 & 0 & 0 & 0 \\
1 & 2 & 1 & 1 & 1 & 1 & 1 \\
1 & 2 & 3 & 3 & 3 & 3 & 3 \\
1 & 2 & 3 & 2 & 2 & 2 & 2 \\
1 & 2 & 3 & 4 & 3 & 3 & 3 \\
1 & 2 & 3 & 4 & 5 & 5 & 4
\end{array}\right], \quad B=\left[\begin{array}{rrrrrrr}
-2 & -2 & -2 & -2 & -2 & -2 & -2 \\
2 & -1 & -1 & -1 & -1 & -1 & -1 \\
2 & 5 & 5 & 5 & 5 & 5 & 5 \\
2 & 5 & 5 & 4 & 4 & 4 & 4 \\
2 & 5 & 5 & 6 & 5 & 5 & 5 \\
2 & 5 & 5 & 6 & 7 & 7 & 7 \\
2 & 5 & 5 & 6 & 7 & 6 & 6
\end{array}\right] .
$$

The matrices are built in such way that the KCF of the pencil contains blocks of all four possible types, $\operatorname{nrank}(A, B)=6$ and the pencil is singular. Its KCF has blocks $J_{1}(1 / 2), J_{1}(1 / 3), N_{1}, L_{1}$, and $L_{2}^{T}$. If we apply Algorithm 1, we get the values in the following table. Note that values $\lambda_{k}$ in the first column are values from Line 8 of Algorithm 1, which are scaled back to match the eigenvalues of the original matrix pencil $A-\lambda B$ whose matrices are scaled in Line 1 since they do not satisfy $\|A\|_{1}=\|B\|_{1}=1$.

\begin{tabular}{c|clll}
\hline$k$ & $\lambda_{k}$ & \multicolumn{1}{|c}{$\left|s_{k}\right|$} & $\left\|V^{*} x_{k}\right\|$ & $\left\|U^{*} y_{k}\right\|$ \\
\hline 1 & 0.333333 & $1.5 \cdot 10^{-2}$ & $1.3 \cdot 10^{-15}$ & $1.3 \cdot 10^{-14}$ \\
2 & 0.500000 & $9.5 \cdot 10^{-4}$ & $1.3 \cdot 10^{-14}$ & $1.9 \cdot 10^{-14}$ \\
3 & $\infty$ & $3.8 \cdot 10^{-19}$ & $2.8 \cdot 10^{-15}$ & $1.3 \cdot 10^{-14}$ \\
4 & $-0.244794+0.421723 i$ & $7.8 \cdot 10^{-3}$ & $5.8 \cdot 10^{-2}$ & $5.6 \cdot 10^{-15}$ \\
5 & $-0.244794-0.421723 i$ & $7.8 \cdot 10^{-3}$ & $5.8 \cdot 10^{-2}$ & $5.6 \cdot 10^{-15}$ \\
6 & 0.383682 & $2.1 \cdot 10^{-4}$ & $2.6 \cdot 10^{-2}$ & $4.2 \cdot 10^{-1}$ \\
7 & 0.478292 & $2.6 \cdot 10^{-4}$ & $9.2 \cdot 10^{-15}$ & $5.2 \cdot 10^{-1}$ \\
\hline
\end{tabular}

The values in the table follow the pattern from the previous section and it is easy to detect that $\lambda_{1}$ and $\lambda_{2}$ are finite true eigenvalues, $\lambda_{3}$ is a true infinite eigenvalue, $\lambda_{4}, \lambda_{5}$, and $\lambda_{7}$ are random eigenvalues, and $\lambda_{6}$ is the prescribed eigenvalue.

EXAMPLE 6.2. We take example C3 from [12] that comes from control theory and belongs to a set of examples $\mathrm{C} 1, \mathrm{C} 2$, and $\mathrm{C} 3$, where each has successively more ill-conditioned eigenvalues. The pencil has the form

$$
A-\lambda B=\left[\begin{array}{rrrrr}
1 & -2 & 100 & 0 & 0 \\
1 & 0 & -1 & 0 & 0 \\
0 & 0 & 0 & 1 & -75 \\
0 & 0 & 0 & 0 & 2
\end{array}\right]-\lambda\left[\begin{array}{lllll}
0 & 1 & 0 & 0 & 0 \\
0 & 0 & 1 & 0 & 0 \\
0 & 0 & 0 & 1 & 0 \\
0 & 0 & 0 & 0 & 1
\end{array}\right]
$$

Its $\mathrm{KCF}$ contains blocks $L_{2}, J_{1}(1)$, and $J_{1}(2)$. As the pencil is rectangular, we add a zero line to make it square. This adds an $L_{0}^{T}$ block to the KCF. Algorithm 1 returns the following table for $A-\lambda B$, from which the finite true eigenvalues 1 and 2 can be extracted.

\begin{tabular}{c|cccc}
\hline$k$ & $\lambda_{k}$ & $\left|s_{k}\right|$ & $\left\|V^{*} x_{k}\right\|$ & $\left\|U^{*} y_{k}\right\|$ \\
\hline 1 & 1.000000 & $1.2 \cdot 10^{-2}$ & $1.7 \cdot 10^{-15}$ & $1.9 \cdot 10^{-15}$ \\
2 & 2.000000 & $1.2 \cdot 10^{-2}$ & $2.3 \cdot 10^{-15}$ & $1.7 \cdot 10^{-15}$ \\
3 & $-0.693767+1.563033 i$ & $2.1 \cdot 10^{-2}$ & $2.3 \cdot 10^{-15}$ & $5.0 \cdot 10^{-1}$ \\
4 & $-0.693767-1.563033 i$ & $2.1 \cdot 10^{-2}$ & $2.3 \cdot 10^{-15}$ & $5.0 \cdot 10^{-1}$ \\
5 & 78.673901 & $2.8 \cdot 10^{-3}$ & $3.3 \cdot 10^{-1}$ & $6.4 \cdot 10^{-1}$ \\
\hline
\end{tabular}

As in [12] we add some noise and perturb initial $A-\lambda B$ into $\widehat{A}-\lambda \widehat{B}$ by adding $10^{-6} \operatorname{rand}(4,5)$ to $A$ and $B$. True eigenvalues of $\widehat{A}-\lambda \widehat{B}$ can still be extracted by Algorithm 1 if we adjust the parameter $\delta_{1}$. The values we get are in the following table:

\begin{tabular}{r|rccc}
\hline$k$ & \multicolumn{1}{|c}{$\lambda_{k}$} & \multicolumn{1}{c}{$\left|s_{k}\right|$} & $\left\|V^{*} x_{k}\right\|$ & \multicolumn{1}{c}{$\left\|U^{*} y_{k}\right\|$} \\
\hline 1 & 0.999990 & $7.6 \cdot 10^{-3}$ & $2.6 \cdot 10^{-15}$ & $5.2 \cdot 10^{-7}$ \\
2 & 2.000058 & $7.6 \cdot 10^{-3}$ & $2.8 \cdot 10^{-15}$ & $5.6 \cdot 10^{-7}$ \\
3 & 101.850555 & $8.2 \cdot 10^{-4}$ & $1.3 \cdot 10^{-1}$ & $3.7 \cdot 10^{-1}$ \\
4 & -14.308508 & $1.9 \cdot 10^{-2}$ & $5.8 \cdot 10^{-16}$ & $4.5 \cdot 10^{-1}$ \\
5 & 15.734162 & $9.3 \cdot 10^{-3}$ & $1.0 \cdot 10^{-17}$ & $4.7 \cdot 10^{-1}$ \\
\hline
\end{tabular}


ExAmple 6.3. This is an example from [14, Sec. 5], where the staircase algorithm fails to find a regular subspace of proper size under a small random perturbation. We take

$$
A_{1}-\lambda B_{1}=\left[\begin{array}{cccc}
0 & 0 & 1 & 0 \\
0 & 0 & 0 & 1 \\
0 & 0 & 0 & 0
\end{array}\right]-\lambda\left[\begin{array}{cccc}
\delta & 0 & 0 & 0 \\
0 & \delta & 0 & 0 \\
0 & 0 & 1 & 0
\end{array}\right],
$$

where $\delta=1.5 \cdot 10^{-8}$. The KCF structure of the pencil is $J_{2}(0)$ and $L_{1}$ which means that 0 is a double eigenvalue. It is reported in [14] that if we add a random perturbation of size $10^{-14}$ to the pencil, then Guptri reports the regular part $J_{1}(0)$ and we have been able to confirm this using a Matlab implementation of Guptri in [34]. If we enlarge the perturbation to $10^{-11}$, Guptri returns no regular part at all, while Algorithm 1 returns two finite true eigenvalues $\lambda_{1}$ and $\lambda_{2}$ from the following table.

\begin{tabular}{c|clll}
\hline$k$ & $\lambda_{k}$ & \multicolumn{1}{c}{$\left|s_{k}\right|$} & \multicolumn{1}{c}{$\left\|V^{*} x_{k}\right\|$} & $\left\|U^{*} y_{k}\right\|$ \\
\hline 1 & $-1.4306543 \cdot 10^{-3}$ & $1.6 \cdot 10^{-11}$ & $5.5 \cdot 10^{-17}$ & $6.7 \cdot 10^{-10}$ \\
2 & $9.9599790 \cdot 10^{-4}$ & $1.6 \cdot 10^{-11}$ & 0.0 & $6.7 \cdot 10^{-10}$ \\
3 & $-2.2641370 \cdot 10^{7}$ & $5.2 \cdot 10^{-9}$ & $2.9 \cdot 10^{-18}$ & $2.6 \cdot 10^{-6}$ \\
4 & $1.1878888 \cdot 10^{0}$ & $1.6 \cdot 10^{-3}$ & $2.0 \cdot 10^{-1}$ & $7.8 \cdot 10^{-1}$ \\
\hline
\end{tabular}

ExAmple 6.4. We take the singular pencil $\Delta_{1}-\lambda \Delta_{0}$ of size $300 \times 300$ from [33, Ex. 18]. This example is related to two random matrices $A$ and $B$ of size $10 \times 10$ in a way that the true eigenvalues of $\Delta_{1}-\lambda \Delta_{0}$ are exactly the values $\lambda$ such that $A+\lambda B$ has a multiple eigenvalue (see Section 3.2). We know from the properties of the problem that there are 90 such values $\lambda$ and that the $\mathrm{KCF}$ of $\Delta_{1}-\lambda \Delta_{0}$ contains $100 N_{1}$ and 10 left and 10 right singular blocks. The conjecture from [33] is that the singular blocks are $5 L_{4}^{T}, 5 L_{5}^{T}, 5 L_{5}$, and $5 L_{6}$ blocks.

This example is also available as demo_double_eig_mp in toolbox MultiParEig [35. The staircase algorithm in MultiParEig fails to extract the finite regular part of size 90 in double precision, but manages to extract all 90 finite true eigenvalues using quadruple precision and the Multiprecision Computing Toolbox [36]. If we apply Algorithm 1 to $\Delta_{1}-\lambda \Delta_{0}$ in double precision, we get the following values:

\begin{tabular}{c|cccc}
\hline$k$ & $\lambda_{k}$ & $\left|s_{k}\right|$ & $\left\|V^{*} x_{k}\right\|$ & $\left\|U^{*} y_{k}\right\|$ \\
\hline 1 & $0.508999+2.016378 i$ & $3.0 \cdot 10^{-3}$ & $2.3 \cdot 10^{-14}$ & $1.6 \cdot 10^{-14}$ \\
$\vdots$ & $\vdots$ & $\vdots$ & $\vdots$ & $\vdots$ \\
89 & $4.266290-0.925962 i$ & $1.4 \cdot 10^{-6}$ & $2.6 \cdot 10^{-13}$ & $7.2 \cdot 10^{-14}$ \\
90 & -0.628208 & $3.2 \cdot 10^{-7}$ & $2.8 \cdot 10^{-14}$ & $1.3 \cdot 10^{-11}$ \\
91 & $\infty$ & $1.1 \cdot 10^{-17}$ & $7.1 \cdot 10^{-15}$ & $7.1 \cdot 10^{-15}$ \\
$\vdots$ & $\vdots$ & $\vdots$ & $\vdots$ & $\vdots$ \\
190 & $\infty$ & $2.8 \cdot 10^{-21}$ & $5.9 \cdot 10^{-15}$ & $7.9 \cdot 10^{-15}$ \\
191 & -6.276934 & $3.2 \cdot 10^{-7}$ & $2.7 \cdot 10^{-14}$ & $4.5 \cdot 10^{-5}$ \\
$\vdots$ & $\vdots$ & $\vdots$ & $\vdots$ & $\vdots$ \\
300 & 7.125982 & $2.3 \cdot 10^{-5}$ & $1.7 \cdot 10^{-1}$ & $1.0 \cdot 10^{-2}$ \\
\hline
\end{tabular}

From the columns $\left\|V^{*} x_{k}\right\|$ and $\left\|U^{*} y_{k}\right\|$ we get $\max _{k=1, \ldots, 190}\left(\max \left(\left\|V^{*} x_{k}\right\|,\left\|U^{*} y_{k}\right\|\right)\right)=1.3 \cdot 10^{-11}$ and $\min _{k=191, \ldots, 300} \max \left(\left\|V^{*} x_{k}\right\|,\left\|U^{*} y_{k}\right\|\right)=4.5 \cdot 10^{-5}$, which shows a clear gap which separates true eigenvalues from the prescribed and random ones. Next, in the set of true eigenvalues there is also a clear gap between $s_{90}$ and $s_{91}$ which separates finite true eigenvalues from infinite ones, since $\min _{k=1, \ldots, 90}\left|s_{k}\right|=3.2 \cdot 10^{-7}$ and $\max _{k=91, \ldots, 190}\left|s_{k}\right|=1.1 \cdot 10^{-17}$.

7. The singular two-parameter eigenvalue problem. We now expand on Section 3.3 . In a two-parameter eigenvalue problem (2EP) [1] we have the equations

$$
\begin{aligned}
& \left(A_{1}+\lambda B_{1}+\mu C_{1}\right) x_{1}=0, \\
& \left(A_{2}+\lambda B_{2}+\mu C_{2}\right) x_{2}=0,
\end{aligned}
$$


where $A_{1}, B_{1}$, and $C_{1}$ are of size $n_{1} \times n_{1}$, and $A_{2}, B_{2}$, and $C_{2}$ are of size $n_{2} \times n_{2}$. Sought are scalars $\lambda, \mu$ and nonzero vectors $x_{1}$ and $x_{2}$ such that (7.1) is satisfied. We say that $(\lambda, \mu)$ is an eigenvalue of the $2 \mathrm{EP}$ and the tensor product $x_{1} \otimes x_{2}$ is the corresponding eigenvector. Define the operator determinants

$$
\begin{aligned}
& \Delta_{0}=B_{1} \otimes C_{2}-C_{1} \otimes B_{2}, \\
& \Delta_{1}=C_{1} \otimes A_{2}-A_{1} \otimes C_{2}, \\
& \Delta_{2}=A_{1} \otimes B_{2}-B_{1} \otimes A_{2} .
\end{aligned}
$$

Then problem (7.1) is related to a coupled pair of GEPs

$$
\begin{aligned}
& \Delta_{1} z=\lambda \Delta_{0} z, \\
& \Delta_{2} z=\mu \Delta_{0} z
\end{aligned}
$$

for a decomposable tensor $z=x_{1} \otimes x_{2}$. If $\Delta_{0}$ is nonsingular, then Atkinson [1] shows that the solutions of (7.1) and (7.3) agree and the matrices $\Delta_{0}^{-1} \Delta_{1}$ and $\Delta_{0}^{-1} \Delta_{2}$ commute. In the nonsingular case the 2EP (17.1) has $n_{1} n_{2}$ eigenvalues and it can be solved with a variant of the QZ algorithm on (7.3); see [18].

It turns out that for many problems occurring in practice both pencils $\left(\Delta_{1}, \Delta_{0}\right)$ and $\left(\Delta_{2}, \Delta_{0}\right)$ are singular and we have a singular 2EP [31. Applications include delay-differential equations [20], quadratic two-parameter eigenvalue problems [32, 19], model updating [5], and roots of systems of bivariate polynomials [37, 2].

The eigenvalues of a singular 2EP (7.1) are the finite regular eigenvalues of (7.3); see Section 3.3. There exists a staircase type algorithm that works on both singular pencils (7.3) simultaneously and extracts finite regular eigenvalues; see [32] and an implementation in [35. However, as illustrated in Examples 6.3 and 6.4, a staircase algorithm may fail. In this section we propose an alternative method that may be applied to a singular 2EP, which in some cases finds finite regular eigenvalues when the staircase algorithm fails, while in some other cases the situation is exactly the opposite.

We can apply Algorithm 1 to $\Delta_{1} z=\lambda \Delta_{0} z$, one of the two singular pencils in (7.3), to compute the $\lambda_{i}$ components of eigenvalues $\left(\lambda_{i}, \mu_{i}\right)$. This is, however, only half of the required information and for each $\lambda_{i}$ we have to find the corresponding $\mu_{i}$. Subsequently, we insert $\lambda=\lambda_{i}$ into (7.1) and search for common eigenvalues $\mu$ of a pair of pencils $\left(A_{1}-\lambda_{i} B_{1}\right)-\mu C_{1}$ and $\left(A_{2}-\lambda_{i} B_{2}\right)-\mu C_{2}$ that may be singular as well. We detect the common eigenvalues by comparing the sets of computed eigenvalues for the first and the second pencil, for which we use Algorithm 1 again. The overall method is given in Algorithm 2.

\section{Algorithm 2: Computing finite regular eigenvalues of a singular 2EP}

Input: Matrices $A_{1}, B_{1}, C_{1}, A_{2}, B_{2}, C_{2}$ from (7.1) which provide $\Delta_{1}$ and $\Delta_{0}$ from (7.2); threshold $\delta$ (default $\delta=\varepsilon^{1 / 2}$ ), and parameters for Algorithm 1 .

Output: Finite regular eigenvalues of (7.1).

1: Compute finite eigenvalues $\lambda_{1}, \ldots, \lambda_{r}$ of $\Delta_{1}-\lambda \Delta_{0}$ using Algo. 1.

2: for $j=1, \ldots, r$

3: $\quad$ Compute eigenvalues $\mu_{1}^{(1)}, \ldots, \mu_{m_{1}}^{(1)}$ of $\left(A_{1}-\lambda_{j} B_{1}\right)-\mu C_{1}$ using Algo. 1 .

4: $\quad$ Compute eigenvalues $\mu_{1}^{(2)}, \ldots, \mu_{m_{2}}^{(2)}$ of $\left(A_{2}-\lambda_{j} B_{2}\right)-\mu C_{2}$ using Algo. 1.

5: $\quad$ Reorder eigenpairs: $\left|\mu_{1}^{(1)}-\mu_{1}^{(2)}\right| \leq \cdots \leq\left|\mu_{m}^{(1)}-\mu_{m}^{(2)}\right|$ for $m=\min \left(m_{1}, m_{2}\right)$.

6: $\quad$ for $k=1, \ldots, m$

7: $\quad$ if $\left|\mu_{k}^{(1)}-\mu_{k}^{(2)}\right|<\delta$ then add $\left(\lambda_{j}, \frac{1}{2}\left(\mu_{k}^{(1)}+\mu_{k}^{(2)}\right)\right)$ to list of eigenvalues. 
Some remarks about Algorithm 2 are in order.

- If we know that each eigenvalue has a unique $\lambda$ component, then we can replace Lines 6 and 7 by selecting $\left(\lambda_{j}, \frac{1}{2}\left(\mu_{1}^{(1)}+\mu_{1}^{(2)}\right)\right)$ regardless of the difference $\left|\mu_{1}^{(1)}-\mu_{1}^{(2)}\right|$.

- If $n_{1}=n_{2}=n$ then the complexity of Line 1 is $\mathcal{O}\left(n^{6}\right)$ while the complexity of Lines 2 to 7 is at most $\mathcal{O}\left(n^{5}\right)$ in case $r=\mathcal{O}\left(n^{2}\right)$.

ExAmple 7.1. Consider a system of bivariate polynomials (cf. [37, Exs. 5.3, 6.2, 6.4])

$$
\begin{aligned}
& p_{1}(\lambda, \mu)=1+2 \lambda+3 \lambda+4 \lambda^{2}+5 \lambda \mu+6 \mu^{2}+7 \lambda^{3}+8 \lambda^{2} \mu+9 \lambda \mu^{2}+10 \mu^{3}=0, \\
& p_{2}(\lambda, \mu)=10+9 \lambda+8 \mu+7 \lambda^{2}+6 \lambda \mu+5 \mu^{2}+4 \lambda^{3}+3 \lambda^{2} \mu+2 \lambda \mu^{2}+\mu^{3}=0 .
\end{aligned}
$$

Using a uniform determinantal representation from [2], we write the above system as a $2 \mathrm{EP}$ of the form

$$
\begin{aligned}
A_{1}+\lambda B_{1}+\mu C_{1} & =\left[\begin{array}{ccccc}
0 & 0 & 4+7 \lambda & 1 & 0 \\
0 & 5+8 \lambda & 2 & -\lambda & 1 \\
6+9 \lambda+10 \mu & 3 & 1 & 0 & -\lambda \\
1 & -\mu & 0 & 0 & 0 \\
0 & 1 & -\mu & 0 & 0
\end{array}\right], \\
A_{2}+\lambda B_{2}+\mu C_{2} & =\left[\begin{array}{ccccc}
0 & 0 & 7+4 \lambda & 1 & 0 \\
0 & 6+3 \lambda & 9 & -\lambda & 1 \\
5+2 \lambda+\mu & 8 & 10 & 0 & -\lambda \\
1 & -\mu & 0 & 0 & 0 \\
0 & 1 & -\mu & 0 & 0
\end{array}\right],
\end{aligned}
$$

where $p_{i}(\lambda, \mu)=\operatorname{det}\left(A_{i}+\lambda B_{i}+\mu C_{i}\right)$ for $i=1,2$. The obtained $2 \mathrm{EP}$ is singular and has 9 regular eigenvalues $\left(\lambda_{j}, \mu_{j}\right)$ which are exactly the 9 solutions of the initial polynomial system.

If we apply Algorithm 2 to the above problem, we get all 9 solutions. In Line 2 we compute first components $\lambda_{1}, \ldots, \lambda_{9}$ as finite eigenvalues of the corresponding singular pencil $\Delta_{1}-\lambda \Delta_{0}$ from (7.3), whose KCF contains $4 L_{0}, 4 L_{0}^{T}, 2 N_{4}, 1 N_{2}, 2 N_{1}$, and $9 J_{1}$ blocks. For each $\lambda_{j}$ we compute the candidates for $\mu_{j}$ in Lines 4 and 5 , where the KCF of singular pencils $\left(A_{i}-\lambda_{j} B_{i}\right)-\mu C_{i}$ contains $1 N_{2}$ and $3 J_{1}$ blocks for $i=1,2$ and $j=1, \ldots, 9$.

We remark that the above approach might also fail, in particular if we apply it to systems of bivariate polynomials of high degree. Some of the eigenvalues of $\Delta_{1}-\lambda \Delta_{0}$ might be so ill-conditioned that the algorithm cannot separate them from the infinite eigenvalues. In such a case a possible solution would be to apply computation in higher precision, using, e.g., the Multiprecision Computing Toolbox [36].

8. Conclusions. We have proposed a method to approximate the finite eigenvalues of a singular pencil by means of a rank-completing perturbation. The use of such a perturbation ensures that, generically, the finite and infinite eigenvalues remain fixed, while there appear newly generated eigenvalues. For many problems we can well distinguish the original eigenvalues from the newly created ones by considering the angles of the eigenvectors with respect to the perturbation spaces, and at the condition numbers of the eigenvalues. Thus, this method may be useful for a wide range of applications.

The proposed method could be an alternative to the class of staircase algorithms, such as e.g., Guptri [17] or a staircase type algorithm for singular two-parameter eigenvalue problems [32] in [35]. These methods can be rapid and accurate, however, the key part of staircase techniques are a number of rank decisions, which can be difficult and ill-posed, see e.g., [14 and Examples 6.3 and 6.4. In some cases, when these methods fail to return even a single eigenvalue, the newly proposed method may still compute all or at least some of the eigenvalues.

A code for the approach developed in this paper is available in [35]. 
Acknowledgments: The authors would like to thank Stefan Johansson for providing a beta version of Matrix Canonical Structure (MCS) Toolbox [34] which includes a Matlab implementation of Guptri and is an important alternative to the original Guptri [17] that we can no longer use in Matlab due to the 32-bit limitation. Furthermore, the authors would like to warmly thank two anonymous referees for their careful reading and many expert suggestions and comments on a previous version of this paper.

Genealogical acknowledgment: During this research project the first two authors found out that they are twelfth cousins. Christian and Michiel thank their common ancestors Caspar Hölterhoff (1552-1625) and Catharina Teschemacher (ca. 1555-1639) for making this possible.

\section{REFERENCES}

[1] F. V. Atkinson, Multiparameter Eigenvalue Problems, Academic Press, New York, 1972.

[2] A. Boralevi, J. van Doornmalen, J. Draisma, M. E. Hochstenbach, and B. Plestenjak, Uniform determinantal representations, SIAM J. Appl. Algebra Geometry, 1 (2017), pp. 415-441.

[3] P. Benner, P. Losse, V. Mehrmann, And M. Voigt, Numerical Linear Algebra Methods for Linear Differential-Algebraic Equations. In: A. Ilchmann, T. Reis (eds), Surveys in Differential-Elgebraic Equations III. Differ.-Algebr. Equa. Forum, pp. 117-175, Springer, Cham, 2015.

[4] R. Byers, C. He, And V. Mehrmann, Where is the nearest non-regular pencil?, Linear Algebra Appl., 285 (1998), pp. 81-105.

[5] N. CotTin, Dynamic model updating - a multiparameter eigenvalue problem, Mechanical systems and signal processing, 15 (2001), pp. 649-665.

[6] E.J. Davison And S.H. Wang, Properties and calculation of transmission zeros of linear multivariable systems, Automatica, 10 (1974), pp. 643-658.

[7] F. De TERÁn AND F. M. DopiCO, Low rank perturbation of Kronecker structures without full rank, SIAM J. Matrix Anal. Appl., 29 (2007), pp. 496-529.

[8] — A note on generic Kronecker orbits of matrix pencils with fixed rank, SIAM J. Matrix Anal. Appl., 30 (2008), pp. 491-496.

[9] F. De TerÁn, F. M. Dopico, AND J. Moro, First order spectral perturbation theory of square singular matrix pencils, Linear Algebra Appl., 429 (2008), pp. 548-576.

[10] J. Demmel, Generalized Non-Hermitian Eigenproblems. Section 2.6 in: Z. Bai, J. Demmel, J. Dongarra, A. Ruhe, and H. van der Vorst (eds.), Templates for the Solution of Algebraic Eigenvalue Problems: a practical guide, pp. 28-36, SIAM, Philadelphia, 2000.

[11] J. Demmel And B. KÅgström, Computing stable eigendecompositions of matrix pencils, Linear Algebra Appl., 88/89 (1987), pp. 139-186.

[12] — Accurate solutions of ill-posed problems in control theory, SIAM J. Matrix. Anal. Appl., 9 (1988), pp. $126-145$.

[13] - The generalized Schur decomposition of an arbitrary pencil $A-\lambda B$-robust software with error bounds and applications. Part I: theory and algorithms, ACM Trans. Math. Software, 19 (1993), pp. 160-174.

[14] A. Edelman And Y. MA, Staircase failures explained by orthogonal versal forms, SIAM J. Matrix Anal. Appl., 21 (2000), pp. 1004-1025.

[15] A. Emami-Naeini And P. Van Dooren, Computation of zeros of linear multivariable systems, Automatica 18 (1982), pp. $415-430$.

[16] F.R. Gantmacher, Theory of Matrices. Volumes 1 and 2, Chelsea, New York, 1959.

[17] Guptri, software for singular pencils, www8.cs.umu.se/research/nla/singular_pairs/guptri/

[18] M. E. Hochstenbach, T. Košir, And B. Plestenjak, A Jacobi-Davidson type method for the nonsingular two-parameter eigenvalue problem, SIAM J. Matrix Anal. Appl., 26 (2005), pp. 477-497.

[19] M. E. Hochstenbach, A. Muhič, And B. Plestenjak, On linearizations of the quadratic two-parameter eigenvalue problem, Linear Algebra Appl., 436 (2012), pp. 2725-2743.

[20] E. Jarlebring And M. E. Hochstenbach, Polynomial two-parameter eigenvalue problems and matrix pencil methods for stability of delay-differential equations, Linear Algebra Appl., 431 (2009), pp. 369-380.

[21] E. Jarlebring, S. KvaAl, And W. Michiels, Computing all pairs $(\lambda, \mu)$ such that $\lambda$ is a double eigenvalue of $A+\mu B$, SIAM J. Matrix Anal. Appl., 32 (2011), pp. 902-927.

[22] B. KÅgström, Singular matrix pencils. Section 8.7 in: Z. Bai, J. Demmel, J. Dongarra, A. Ruhe, and H. van der Vorst (eds), Templates for the Solution of Algebraic Eigenvalue Problems: a practical guide, pp. 260-277, SIAM, Philadelphia, 2000.

[23] P. Kunkel and V. Mehrmann, Differential-Algebraic Equations: Analysis and Numerical Solution, EMS 
Publishing House, Zürich, Switzerland, 2006.

[24] A.J. Laub AND B.C. Moore, Calculation of transmission zeros using QZ techniques, Automatica, 14 (1978), pp. $557-566$.

[25] D. S. Mackey, N. Mackey, C. Mehl, and V. Mehrmann, Skew-symmetric matrix polynomials and their Smith forms, Linear Algebra Appl., 438 (2013), pp. 4625-4653.

[26] — Möbius transformations of matrix polynomials, Linear Algebra Appl., 470 (2015), pp. 120-184.

[27] C. Mehl, V. Mehrmann, and M. Wojtylak, On the distance to singularity via low rank perturbations, Operators and Matrices, 9 (2015), pp. 733-772.

[28] — Parameter-dependent rank-one perturbations of singular Hermitian or symmetric pencils, SIAM J. Matrix Anal. Appl., 38 (2017), pp. 72-95.

[29] - Linear algebra properties of dissipative Hamiltonian descriptor systems, SIAM J. Matrix Anal. Appl., 39 (2018), pp. 1489-1519.

[30] V. Mehrmann, private communication, 2018.

[31] A. Muhič And B. Plestenjak, On the singular two-parameter eigenvalue problem, Electron. J. Linear Algebra, 18 (2009), pp. 420-437.

[32] _ On the quadratic two-parameter eigenvalue problem and its linearization, Linear Algebra Appl., 432 (2010), pp. 2529-2542.

[33] - A method for computing all values $\lambda$ such that $A+\lambda B$ has a multiple eigenvalue, Linear Algebra Appl., 440 (2014), pp. 345-359.

[34] MCS Toolbox. The Matrix Canonical Structure Toolbox for Matlab, www.cs.umu.se/english/research/groups/matrix-computations/stratigraph

[35] MultiParEig. Toolbox for multiparameter eigenvalue problems, www .mathworks.com/matlabcentral/fileexchange/47844-multi

[36] Multiprecision Computing Toolbox. Advanpix, Tokyo. www.advanpix.com

[37] B. Plestenjak And M. E. Hochstenbach, Roots of bivariate polynomial systems via determinantal representations, SIAM J. Sci. Comput., 38 (2016), pp. A765-A788.

[38] N. VALEeV, On a spectral property of irregular pencils, Ufa Mathematical Journal, 4 (2012), pp. 44-52.

[39] - On quasiregular spectrum of matrix pencils, Doklady Mathematics, vol. 88, Springer, 2013, pp. 545-547.

[40] P. VAn DoOREn, The computation of Kronecker's canonical form of a singular pencil, Linear Algebra Appl., 27 (1979), pp. 103-140.

[41] —, Reducing subspaces: Definitions, properties and algorithms. In: B. Kågström, A. Ruhe (eds.), Matrix Pencils. Lecture Notes in Mathematics, vol. 973. Springer, Berlin, Heidelberg, 1983, pp. 58-73.

[42] J.H. Wilkinson, The Algebraic Eigenvalue Problem, Oxford University Press, Oxford, 1965.

[43] - Kronecker's canonical form and the QZ algorithm, Linear Algebra Appl., 28 (1979), pp. $285-303$. 